\title{
Dynamic signatures of lipid droplets as new markers to quantify cellular metabolic changes
}

\author{
Chi Zhang ${ }^{1}$ and Stephen A. Boppart * 1, 2, 3, 4,5 \\ ${ }^{1}$ Beckman Institute for Advanced Science and Technology \\ ${ }^{2}$ Department of Bioengineering \\ ${ }^{3}$ Department of Electrical and Computer Engineering \\ ${ }^{4}$ Carle Illinois College of Medicine \\ ${ }^{5}$ Cancer Center at Illinois \\ University of Illinois Urbana Champaign
}

*Stephen A. Boppart, boppart@illinois.edu

\begin{abstract}
The metabolic properties of live cells are very susceptible to intra- or extra-cellular perturbations, making their measurements challenging tasks. We show that the dynamics of lipid droplets (LDs) carry information to measure the lipid metabolism of live cells. Coherent anti-Stokes Raman scattering microscopy was used to statistically quantify LD dynamics in living cells in a label-free manner. We introduce dynamic signatures of cells derived from the LD displacement, speed, travel length, and directionality, which allows for the detection of cellular changes induced by stimuli such as fluorescent labeling, temperature change, starvation, and chemical treatment. Histogram fittings of the dynamic signatures using lognormal distribution functions provide quantification of changes in cellular metabolic states. The LD dynamics also enable separation of subpopulations of LDs correlated with different functions. We demonstrate that LD dynamics are new markers to quantify the metabolic changes in live cells.
\end{abstract}




\section{Main Text \\ Introduction}

Metabolic homeostasis is the key to maintain proper functions of living cells, while altered metabolism is usually associated with disease transitions. Among different metabolites, lipids are crucial for cellular energy storage, membrane construction, signaling, and hormone production. Lipid droplets (LDs), a key organelle that is responsible for the storage and regulation of neutral lipids in cells, has stepped into the spotlight of lipid research in the past two decades due to its direct association with cancer transition and progression (Bozza and Viola, 2010; Petan et al., 2018; Tirinato et al., 2017). Scientists realized that LDs are not simple and inert lipid deposits, but are highly dynamic and functional structures which are major lipid regulators in cells (Baenke et al., 2013; Walther and Farese Jr, 2012). Recent studies found that LD biogenesis is directly linked to cellular stress factors such as reactive oxygen species (Bailey et al., 2015; Welte, 2015) and hypoxia (Biron-Shental et al., 2007; Koizume and Miyagi, 2016), and offers protective effects for cells in stress environments (Bosma et al., 2014; Herms et al., 2015; Jarc et al., 2018). This highlights the critical roles of LDs in regulating cell metabolism to maintain cellular homeostasis under various external stimuli. Besides, LDs were found to accumulate in various cancer cells (Mitra et al., 2017; Morjani et al., 2001), suggesting their indispensable association with metabolic reprogramming in cancer. LDs were reported to protect cancer cells from the treatment of chemotherapeutic drugs (Cotte et al., 2018) and accumulate cholesteryl ester to promote tumor growth (Yue et al., 2014). Elucidating LD metabolism has led to discoveries of new targets for cancer treatment (Liu et al., 2017; Yue et al., 2014) and cancer stem cell suppression (Li et al., 2017).

Despite numerous efforts that have investigated LD content, size, and amount associated with various stimuli and disease transitions, our understanding of LD dynamics remains primitive. Especially, the dynamic information of LDs, which is an important signature to probe cell metabolism, is largely underexplored due to the lack of appropriate techniques. To identify and distinguish LDs from other organelles, chemical labeling is typically a requisite. Different from other parameters, the dynamic information can only be probed in living samples, ruling out many widely used labeling and imaging techniques such as immune-fluorescence imaging and electron microscopy. Lipophilic dyes such as BODIPY can label LDs in live cells. However, LD dynamics are very sensitive and susceptible to cellular functions, which are perturbed by the introduction of exogenous fluorescent probes. A label-free microscope with lipid selectivity is the desired tool to measure LD dynamics. Mid-infrared and Raman spectroscopic imaging, which are based on vibrational absorption and scattering, have been used to image lipid contents in cells (Pleitez et al., 2020; Zhang et al., 2016). Mid-IR imaging cannot compete with Raman imaging in terms of penetration depth and spatial resolution (Cheng and Xie, 2015), while spontaneous Raman imaging typically has much slower imaging speed on the order of minutes to hours, which is too slow to capture dynamic signatures of LDs in living cells (Palonpon et al., 2013). Coherent Raman scattering microscopy has offered new ways to probe LDs in living cells in a label-free manner with sub-micron resolution and fast imaging speed (Camp Jr et al., 2014; Cheng et al., 2002; Di Napoli et al., 2014; Evans et al., 2005; Freudiger et al., 2008; Fu, 2017; Fu et al., 2012; He et al., 2017; Liao et al., 2015; Lu et al., 2015; Min et al., 2011; Ozeki et al., 2012; Potma et al., 2002; Slipchenko et al., 2009; Wang et al., 2013; Zhang et al., 2013; Zumbusch et al., 1999). With this approach, LD dynamics can be visualized in real-time (Bradley et al., 2016; Nan et al., 2006; Rinia et al., 2008; Zhang et al., 2017).

Although some initial studies have been performed using coherent anti-Stokes Raman scattering (CARS) (Nan et al., 2006) or stimulated Raman scattering (SRS) to measure LD dynamics (Zhang et al., 2017), the comparison of LD trafficking under different conditions is largely qualitative and preliminary. In a previous study, results show a correlation between LD displacement and 
lipogenesis (Zhang et al., 2017). However, quantitative ways to measure and compare LD dynamics under various conditions are still lacking, preventing the systematic understanding of LD dynamics associated with different environmental stimuli and drug treatment. In this study, we introduce dynamic signatures of living cells by quantification of LD displacement, speed, travel length, and directionality using CARS microscopy. We also used lognormal distributions to model the statistics of LD dynamics, which allows for a quantitative comparison of changes in LD dynamics related to stimuli and drug treatment. We systematically studied LD dynamic responses to fluorescent labeling, hypothermia exposure, apoptosis, starvation, adenosine monophosphate activated protein kinase (AMPK) activation, and drug treatments targeting different lipid metabolic enzymes. We found reduced directionality of LD trafficking during hypothermia exposure and apoptosis, and changes in the ratio of synthesis- and degradation-related LDs during starvation, AMPK activation, and fatty-acid-related enzyme inhibition. Collectively, LD dynamics captured and quantified by CARS imaging offer the potential for a better understanding of biological processes. The LD dynamics might also function as new biomarkers for cellular responses to disease transitions and drug treatments. Our approach allows biologists to understand real-time cell metabolism from a novel angle, and would have important applications in drug development and cancer research.

\section{Results}

\section{Quantification of LD dynamics}

CARS microscopy was used to image LDs in living cells. We tuned the pump and Stokes beams to excite the lipid $\mathrm{CH}_{2}$ vibration centered at $2884 \mathrm{~cm}^{-1}$. The lab-built CARS microscope used in this study was pumped by a dual-output pulsed laser source and constructed based on an upright microscope frame (Figure S1). The details of our CARS microscope can be found in Materials and Methods. Briefly, the pump and Stokes beams at the sample were $\sim 14 \mathrm{~mW}, 1 \mathrm{ps}$, and $\sim 10$ $\mathrm{mW}, 2 \mathrm{ps}$, respectively. CARS signals were collected in the transmission direction (Figure 1A). Two-photon excitation fluorescence (TPEF) signals from the sample can be simultaneously acquired in the epi direction if needed. The pixel dwell time used for imaging was $10 \mu \mathrm{s}$, and the imaging speed for images with $400 \times 400$ pixels was $2.2 \mathrm{~s} /$ frame. A 100 -frame image stack was acquired for each measurement and multiple regions were imaged for each condition (Figure 1B). A Particle Tracker ImageJ Plugin (Sbalzarini and Koumoutsakos, 2005) was used to trace the trajectories of individual LDs that were detected in more than 20 frames. A sample LD trajectory is displayed in Figure $1 \mathrm{C}$ and Video S1. A MATLAB-based lab-written program was used to quantitatively calculate all the essential parameters of the trajectories used for statistical analysis.

Three parameters that can be further used to quantify the LD dynamics were directly derived from the trajectories, including the maxd, the speed, and the length. The maxd value is defined as the maximum displacement of the LD movement during the time of image acquisition. Different from a previous study which only measured the maximum displacement from the starting point (Zhang et al., 2017), we take the maximum value of the maximum displacement starting from all frames as the maxd value (Figure S2). This is a more valid way to quantify the maximum relocation distance of LDs within the time of measurement. The value length is the total trajectory length, and the value speed is defined as length divided by the total time of the trajectory.

The LD trajectories can be plotted in the maxd-speed-length 3D space as a signature to characterize LD dynamics of living cells. Figures 1D,E plot the 3D LD dynamic signature of MIAPaCa-2 pancreatic cancer cells (brown dots) at $37^{\circ} \mathrm{C}$ in Dulbecco's Modified Eagle Medium (DMEM) $((+)$ glucose $)+10 \%$ fetal bovine serum (FBS) $+1 \%$ penicillin-streptomycin antibiotics. The projection of this 3D dynamic signature onto the three 2D planes (black dots), including speed-maxd, maxd-length, and length-speed, can be used to better display and compare the changes in LD dynamics. Density contour plots of the dynamic signature in the three planes are shown in Figure 1F, which highlight the population density of the trajectories. Further, the 
projections of the dynamic signature on the maxd, speed, and length axes (Figures 1G-I) reveal histograms of the trajectories and can be compared quantitatively through the mathematical fitting. We found that the lognormal function is the best fitting function to quantify these histograms (Figures 1G-I).

In addition to the three parameters defined above, we also derived a 'directional factor (DF)' to quantify the directionality of the trajectories. The $D F$ is defined as the total trajectory length divided by the maxd. A more directional LD trajectory will have a smaller $D F$ value. We plotted the MIAPaCa-2 LD dynamics in the DF-maxd plane and found a reciprocal relationship between the two parameters (Figure 1J). This indicates that the LDs having longer maxd tend to be more directional in their movement, while the LDs having a shorter maxd were less directional. This can be explained by the two types of LD dynamics in living cells: LDs traveling in the cytosol are transported along microtubules and are more directional with a longer displacement; while LDs bound to the endoplasmic reticulum $(E R)$ have less freedom to move and are much less directional (Zhang et al., 2017).

Figure S3 plots 3D and 2D dynamic signatures of the H358 lung cancer cell line and the MDAMB-231 breast cancer cell line, respectively, under normal culture conditions. Comparing these cell lines with MIAPaCa-2 cells, we found that different cell lines have different dynamic signatures. The value of length/speed is proportional to the number of imaging frames that the LD trajectories occupy. Two populations are identified in the MIAPaCa-2 length-speed graph (Figure S4A). The LDs appeared in higher frame numbers (red gate in Figure S4A) are less likely to move out of the focal plane and tend to have a slower speed (Figure $1 \mathrm{~K}$, and Figure S4). The evidence suggests that LDs forming this population are more likely associated with ER and lipid synthesis. Such a conclusion was further verified by comparing the 2D dynamic signatures in the length-speed domain (Figure S5) during starvation which is known to reduce LD synthesis and increase LD degradation. $\mathrm{H} 358$ cells have a higher percentage of LDs associated with this population (Figure S3C), implying a different LD metabolic rate in H358 cells compared to MIAPaCa-2 cells.

We can gate subpopulations of the trajectories in the DF-maxd domain and further explore the speed and length features. We gated the trajectories having $D F>10$ (red) and maxd >3 (blue) (Figure 1J) and plotted the speed and length values for both populations. The results indicate that less directional LDs tend to have a lower speed and a shorter length (Figures $1 \mathrm{~K}, \mathrm{~L})$. These features unveil the dynamic characteristics of different types of LDs in cells.

The quantification methods developed here allow us to investigate LD dynamics and cellular lipid metabolism from a novel angle. We subsequently explored the responses of LD dynamics of living cells in response to various stimuli.

\section{Staining perturbations to LD dynamics}

Lipophilic dyes are commonly used to visualize LDs in living cells. We compared LD dynamic signatures using our hybrid CARS-TPEF microscope for living cells with and without fluorescent labeling. BODIPY green was used to label LDs in MIAPaCa-2 cells and CARS/TPEF time-lapse image stacks were acquired to quantify the LD dynamics. Compared to the non-labeled control group, BODIPY labeling groups with $2 \mu \mathrm{g} / \mathrm{mL}$ and $4 \mu \mathrm{g} / \mathrm{mL}$ dye concentration for $1 \mathrm{hr}$, as well as with $2 \mu \mathrm{g} / \mathrm{mL}$ for $6 \mathrm{hr}$, show clear shifts of $D F$ towards higher values and shifts of maxd towards lower values (Figure S6). We fit the maxd and DF values for all four cases (Figure S7A,C) and compared the derived $X_{C}$ value which is associated with the median value of the curves. The results clearly show decreased $X_{c}$ values for two of the labeled groups in the maxd domain and increased $X_{C}$ values for all labeled groups in the $D F$ domain (Figure S7B,D). A 2D plot of $X_{C}$ values in $D F$ and maxd domains of all four cases allows us to better distinguish the staininginduced change of LD dynamics (Figure S7E). Further, we can take the ratio of LDs having DF> 
10 and maxd $>3 \mu \mathrm{m}$, and the ratio of LDs having maxd $<2 \mu \mathrm{m}$ and $D F<5$, both of which show clear increases for the all three labeled cases (Figure S7F). These changes indicate LDs tend to reduce their movement, directionality, and relocation distance when BODIPY is used. This cellular perturbation suggests that label-free imaging using CARS microscopy is a more appropriate way to quantify LD dynamics than using fluorescent dyes.

From the CARS images, we discovered that the $6 \mathrm{hr}$ treatment of MIAPaCa-2 cells with $2 \mu \mathrm{g} / \mathrm{mL}$ BODIPY can increase cell membrane blebbing (Figure S8), and $1 \mathrm{hr}$ treatment of MIAPaCa-2 cells with $4 \mu \mathrm{g} / \mathrm{mL}$ can change the morphology of the cells to a more rounded shape (Figure S9), both of which confirmed the functional perturbation of the BODIPY dye to live cells.

\section{Low-temperature effect on LD dynamics}

In addition to the perturbation due to staining, another common environmental stimulus for live cells is a change in temperature. It is believed that cell metabolism scales with temperature because the enzyme activity is only optimal within a relatively small temperature range. We explore how LD dynamics change with temperature, offering a label-free indicator of cellular response due to temperature change. We first measured the MIAPaCa-2 LD dynamics at $37^{\circ} \mathrm{C}$. Then we turned off the temperature maintaining system and exposed the live cells to room temperature $\left(24^{\circ} \mathrm{C}\right)$ for $1 \mathrm{hr}$ before measurement. Next, we reheated the sample back to $37^{\circ} \mathrm{C}$ and measured the recovering process. The dynamic signature projected onto the DF-maxd domain for the three conditions are shown in Figures $2 \mathrm{~A}-\mathrm{C}$. We found that the $1 \mathrm{hr} 24^{\circ} \mathrm{C}$ exposure tends to shift the LDs towards a higher $D F$ value and a lower maxd value, indicating reduced active transportation and increased non-directional movement impacted by the hypothermia exposure. The maxd and DF histograms of LDs in all conditions, together with the lognormal fitting results, are shown as Figures $2 \mathrm{D}, \mathrm{F}$, respectively. We derive the median-value-associated $X_{C}$ values from the lognormal fitting, and the $\sigma$ values, which represent the skewness of the curves. A comparison of these values (Figures 2E,G) shows that both the median values and the skewness of maxd histograms decreased after $1 \mathrm{hr}$ of $24^{\circ} \mathrm{C}$ exposure, and recovered to the $37^{\circ} \mathrm{C}$ levels when the samples were reheated back to $37^{\circ} \mathrm{C}$. These results indicate that short-term hypothermia exposure of these living cells does not cause irreversible metabolic changes.

Next, we studied the effect of longer hypothermia exposure. The MIAPaCa-2 cells were maintained at $24{ }^{\circ} \mathrm{C}$ for up to $4 \mathrm{hr}$. We measured LD dynamics before hypothermia, at $1 \mathrm{hr}$ exposure, $4 \mathrm{hr}$ exposure, and after the samples were reheated back to $37^{\circ} \mathrm{C}$ after the $4 \mathrm{hr}$ exposure. From the DF-maxd domain plots, as shown in Figure S10, we discovered continuous shifts towards larger DF and smaller maxd values as a function of hypothermia exposure time. The fitting results reveal a decrease in both the $X_{c}$ and the $\sigma$ on the maxd domain (Figures $2 \mathrm{H}, \mathrm{I}$ ), and an increase of these values on the DF domain (Figures $2 \mathrm{~J}, \mathrm{~K}$ ) induced by the low temperature. Interestingly, we found that after reheating the sample back to $37^{\circ} \mathrm{C}$, the LD dynamic signatures did not recover to their original levels (Figure $\mathrm{S} 10$ and Figures $2 \mathrm{H}-\mathrm{K}$ ). These results indicate that a $4 \mathrm{hr}$ hypothermia exposure causes irreversible changes to cell functions or the changes that cannot be reversed in the first hour of recovery.

\section{Drug-induced apoptosis}

Apoptosis is a common and highly regulated process controlling cell death. Analyzing the LD movement, we can better understand this process from a dynamic angle and identify the dynamic signatures indicative of this change. To induce apoptosis, we treated cells with staurosporine (STS) (500 nM final concentration) and measured the LD dynamics as a function of treatment time $(0,1,3,11 \mathrm{hr})$. From the DF-maxd plots, we found that STS treatment tends to shift the LD trajectories towards larger DF and smaller maxd values (Figures 3A-D). Such a change was amplified for longer STS treatment. Histogram fitting in the maxd domain quantifies the continuous 
decrease in both the median value and the skewness of LD trafficking distribution induced by STS and treatment time (Figures 3E,F), which can be correlated in the $X_{c}-\sigma$ space (Figure 3G). Similarly, the analysis in the DF domain shows increases in the median value and the skewness of LD trafficking as a function of treatment time (Figures $3 \mathrm{H}-\mathrm{J}$ ).

Such LD dynamic changes were reproduced by using a higher concentration of STS $(1 \mu \mathrm{M})$. Very similar trends were observed with much-amplified changes at a similar treatment period (Figure S11). The major biological function of STS is binding to protein kinase to prevent adenosine triphosphate (ATP) binding to the same enzyme. It was reported that STS can produce a timedependent and concentration-dependent increase in the caspase-3 activity, which is linked to DNA fragmentation (Belmokhtar et al., 2001; Yue et al., 1998). Studies also showed that STS can alter the mitochondrial membrane potential and causes cytochrome c release (Ahlemeyer et al., 2002; Mirkes and Little, 2000). One of the possible causes of changes in LD dynamics is likely due to the reduction of ATP production because of the STS-induced mitochondria dysfunction. The active transport of LDs, which is usually more directional and with a larger maxd value, is powered by ATP.

We used MIAPaCa-2 cells to perform the majority of the studies. As demonstrated in Figure S3, different cells might have different dynamic signatures. However, they might share similar changes in certain conditions. For example, we compared changes of LD dynamics of H358 cells during hypothermia exposure and starvation (Figure S12) and found very similar trends as the MIAPaCa-2 cells (Figures 2,4). If we compare the dynamic signature changes in this apoptosis study with the previous fluorescent labeling and temperature-dependent studies, we find that all perturbations tend to induce decreases in the directionality and the maxd for LD movements, which are likely common signatures for perturbed, unhealthy, and 'unhappy' cells.

\section{Starvation and AMPK activation}

LDs are energy storage reservoirs and regulators in cells. It is therefore critical to understand how energy metabolism is associated with LD dynamics, and if the LD trafficking information can provide additional insights for cell metabolism. We explored metabolic manipulations related to the AMPK pathway, a central signaling pathway of energy homeostasis of cells (Hardie et al., 2012). AMPK pathway can be activated through energy starvation or small activator molecules (Hardie, 2016; Kim et al., 2016; SALT et al., 1998). We first created four conditions to explore the effects of glucose and fatty acids on LD dynamics, including control ((+)glucose, (+)fatty acids), glucose only ((+)glucose, (-)fatty acids), FBS only ((-)glucose, (+)fatty acids), and no glucose no FBS ((-)glucose, (-)fatty acids). MIAPaCa-2 cells were cultured under these conditions and measured at $24 \mathrm{hr}$ and $72 \mathrm{hr}$ time points. We plotted the dynamic signatures of MIAPaCa-2 cells in the speed-maxd domain for all the conditions (Figure S13) and found an increase in the overall speed at $72 \mathrm{hr}$ but not at $24 \mathrm{hr}$ starvation, with both glucose and fatty acids deprived (Figures 4AC). Then we studied the same cells treated with $1 \mathrm{mM} \mathrm{5-aminoimidazole-4-carboxamide-1- \beta -D-}$ ribofuranoside (AICAR), an AMPK agonist and direct activator, in normal and starved culture conditions, and found a similar transition of the dynamic signature in the latter but not in the former condition (Figures 4D,E). Since both starvation and AMPK activation activates the AMPK pathway, which effectively slows down fatty acid synthesis and increases degradation, the very similar trends in Figures 4C,E are likely indicators of AMPK activation. AICAR treatment speeds up such a change in the starved condition since $24 \mathrm{hr}$ starvation by itself does not cause the same change (Figure 4B).

AMPK activation causes reduced LD synthesis and increased LD degradation. Therefore, fewer LDs are associated with the ER, and more are transitioned to the cytosol for degradation and active transportation. From previous studies, we discovered that active transportation tends to have higher speed and maxd values compared to the ER-associated non-directional movement 
(see Figures $1 \mathrm{~J}, \mathrm{~K}$ ). Consequently, the metabolic signature change in the speed-maxd domain associated with AMPK activation is the increase in the speed and maxd values.

If we compare the AICAR treatment group under the normal condition (Figure 4D) with the nontreated group (Figure 4A), we found a decrease in the LD speed. This indicates AICAR treatment of MIAPaCa-2 cells without starvation is causing an opposite change to LD dynamics compared to the AMPK activation. Such a change implies an increased LD synthesis/degradation ratio. MTT cell proliferation assay results show that MIAPaCa-2 cells are experiencing an increased proliferation after being treated by the AICAR in normal culture media (Figure S14). This indicates the continued growth of the cells, which can be correlated with the increased LD synthesis.

To further understand the changes in LD dynamics, we plot the histograms of LD trajectories on the speed axis for quantitative comparison. We found that a single lognormal distribution function cannot achieve good fitting of the histogram (Figure 4F, upper panel). This is because subpopulations of LDs in the cells are better separated in the speed domain. Using a duallognormal function, we can better fit the speed histogram (Figure 4F, bottom panel) and distinguish the two LD populations having different speed distributions. We fit the MIAPaCa-2 speed histogram for all the five conditions (Figure 4G) and plot the amplitude (A) values, which represent the fractions of the LDs in each population, over $X_{c}$, which associates with the median speed of the population (Figure $4 \mathrm{H}$ ). We found that for all conditions, there are two pools of LDs, one $\left(A_{1}\right)$ centered around $0.05 \mu \mathrm{m} / \mathrm{s}$ with the other $\left(A_{2}\right)$ around $0.1 \mu \mathrm{m} / \mathrm{s}$. $A_{1}$ is the population having small speed and maxd values, thus associated with ER and LD synthesis; while $A_{2}$ is the population having larger speed and maxd values, thus associated with cytosol and LD degradation. If we take the $A_{1} / A_{2}$ ratio, we find that AICAR treatment under normal conditions has significantly increased this ratio compared to the control, that $24 \mathrm{hr}$ starvation has a similar ratio as the control, while AICAR + starvation for $24 \mathrm{hr}$ and starvation for $72 \mathrm{hr}$ have much-reduced ratios (Figure 4I). These changes reveal the differences in the ratio between the two pools of LDs under different conditions.

We then analyzed the quantity of LDs using CARS images taken from the five conditions. Sample images are shown as Figures 4J-N, from which we can see an obvious increase in the total LD amount for the AICAR treatment group under the normal condition (Figure 4M). We quantify the total LD amount using an intensity thresholding method as displayed in Figure S15, and found that the LD area percentage of the cells for the five conditions has very similar trends as the $A_{1} / A_{2}$ ratio we measured from the dynamic analysis (Figures $4 I, 0$ ). These results indicate that an increased ratio of LDs in the $A_{1}$ group which is associated with LD synthesis is directly linked to a higher amount of total LDs in cells, suggesting a higher rate of LD synthesis which is observed in the AICAR+normal condition group. On the other hand, higher rates of LD degradation and decreased total LD amount are detected from the AICAR+starvation and $72 \mathrm{hr}$ starvation groups, likely caused by the AMPK activation.

If we look at the $24 \mathrm{hr}$ starvation group with only FBS (Figure S13D), we find a decrease of LD speed. We quantify the $A_{1}$ and $A_{2}$ LD groups by fitting the speed histograms in this condition and compare the results with the control group. We discovered an increase in the $A_{1} / A_{2}$ ratio, which indicates increased LD synthesis (Figure S16A-F). From these results, we predict that compared to the control group, fatty acids alone as the energy source would increase the LD synthesis. This conclusion was further confirmed by the analysis of the LD amount using the intensity thresholding method discussed before (Figure S16G-I).

\section{Lipid-metabolism-related enzyme inhibition}

After exploring the AMPK-related effects, we directly perturbed the lipid metabolism by targeting different fatty-acid-regulating enzymes and studied the LD dynamics associated with these 
perturbations. Two compounds were used to inhibit fatty acid degradation: ranolazine, which inhibits thiolase (Kantor et al., 2000; Sabbah et al., 2002), and etomoxir, which inhibits carnitine palmitoyltransferase I (CPTI) (Reaven et al., 1988). One chemical, C75, was used to inhibit fatty acid synthase (FASN) (Kim et al., 2004). Besides, tunicamycin was deployed to induce ER stress (Banerjee et al., 2011). From the dynamic signatures in the speed-maxd domain, we found that the LD speed values of MIAPaCa-2 cells treated by fatty acid degradation inhibitors were much lower than those treated by the ER-stress inducer and the FASN inhibitor (Figures 5A-D). Quantitative histogram fitting on the speed domain provides $A_{1}$ and $A_{2}$ population information for all four treatment groups (Figure $5 E$ ). Comparing with the control group, the $A_{1} / A_{2}$ population ratio (Figure 5F) shows an increased fraction of synthesis-related LDs for thiolase inhibition (ranolazine), similar fractions of synthesis-related LDs for CPT1 inhibition (etomoxir), and reduced fractions of synthesis-related LDs for ER stress (tunicamycin) and FASN inhibition (C75). We also compared the maxd values for all the conditions and found that the $X_{c}$ values of maxd follow the opposite trend as the $A_{1} / A_{2}$ ratio (Figure $5 G$ ). Since $A_{1}$ is associated with $L D$ synthesis and $A_{2}$ is linked to LD degradation, while LD synthesis is correlated with non-directional movement on ER and LD degradation is linked to active transportation, the trends in maxd reflect a similar metabolic change in cells as was unveiled by the $A_{1} / A_{2}$ ratio. We further plotted the $A_{1} / A_{2}$ ratio against the maxd $X_{C}$ value on a 2D graph (Figure $5 \mathrm{H}$ ) and found that increased and reduced LD synthesis processes are diverging to different directions from the control point. Sample CARS images from the four treatment groups are shown in Figures 5I-L. The ER areas of the MIAPaCa-2 cells have higher $\mathrm{CH}_{2} \mathrm{CARS}$ intensities than the cytosol, but lower signal than the LDs. Using intensity thresholding, we can highlight the ER areas from the cells directly from CARS images (Figure S17), which agrees well with the results from ER labeling using ER-Tracker ${ }^{\mathrm{TM}}$ Green (Figure S17). CARS images show that the LD-degradation inhibited groups have more ER-bound LDs compared to the ER-stress and the FASN inhibited groups (see green areas in Figures 5I-L).

We also studied the effect of another thiolase inhibitor, trimetazidine (Figure S18) (Kantor et al., 2000). Interestingly, from the histogram fitting (Figure S18B,C), we found this case does not fall onto the curve of other conditions in the $A_{1} / A_{2}-\operatorname{maxd} X_{c}$ space (Figure S18D), but deviates from the control toward a new direction. Such an abnormal change in LD dynamics indicates something abnormal happened for cell metabolism. We investigated the CARS images and found that trimetazidine tends to induce LD aggregation, as shown in Figure S18E. Many LDs aggregated after $24 \mathrm{hr}$ treatment and did not separate even over $220 \mathrm{~s}$ (Figure S18E and Video S2). These LD aggregates are in the cytosol, resulting in a low $A_{1} / A_{2}$ ratio. Since different $L D$ s are linked to different microtubules and are unlikely to move long distances once aggregated, these assemblies have much less maxd value, bringing down the median value $\left(X_{c}\right)$ of the entire maxd histogram.

\section{Discussion}

For living cells, if an image is their 'ID card', then their dynamics are 'live-videos' of their behaviors. Dynamics provide useful information that cannot be derived from static images. Through the quantification of LD dynamics in real-time using a label-free approach, we derived the speed, displacement, length, and directionality of LD movements in cells, which allowed us to monitor environmental- and stimuli-induced changes in cellular dynamics in real-time. We, for the first time, quantify the dynamic changes by histogram fitting and comparative analysis. Quantification of LD dynamics offers the opportunity to separate different populations of LDs based on their different metabolic processes (LD synthesis and degradation) within cells, and shed new light on the study of lipid metabolism in living cells. The quantitative LD dynamic information, together with label-free imaging, provides scientists with a new method to understand stimuli- and drug-induced metabolic reprogramming in living cells. We expect that the knowledge obtained from these 
organelle dynamics will help biologists to better understand cell metabolism under stress conditions and better analyze cell responses to various drug treatments.

Several factors impact organelle dynamics in living cells. Reduced LD displacement after shortterm hypothermia exposure is most likely induced by the reduction of energy flux, while long-term hypothermia exposure might cause manifold changes in cell metabolism. The impact of STS on LD dynamics is also likely multifarious, including changes in the cellular cytoskeleton, ATP production, and DNA fragmentation. More focused studies are expected to further understand such influences and impacts. Regarding the AMPK activation and lipid metabolism studies, we have observed clear trends in various conditions that were revealed by LD dynamic analysis and can be explained through the changes in the ratio of LD synthesis/degradation. The conclusions obtained from the dynamic analysis also well-correlate with the evidence observed in conventional image-based quantitative analysis. We have therefore extended our understanding of cell metabolism from this novel angle.

We want to point out that there are errors in trajectory tracking and linking, as well as possible false LD particles detected in the experiments. We have used several approaches to minimize the impact of these errors on our conclusions. First, we set a frame number filter to reject trajectories of less than 20 frames. This filtered out the majority of false trajectories caused by intensity variations and spikes in images. We also optimized the particle linking parameters and used the optimal values for the cutoff, link range, and displacement. We noticed that LD dynamic signatures of the same cell line in the same environment might also be different in different days, and therefore measured a control group for each study. The majority of the control groups have quite similar dynamic signatures, though a few of them are slightly different.

Besides the dynamics of LDs, other organelles dynamics might give us more metabolic information. For example, the mitochondrion, which contains a large number of NADH molecules, can be visualized through autofluorescence at 400-500 nm using the TPEF modality. Therefore, CARS/TPEF dual-channel imaging can simultaneously quantify the dynamics of both LDs and mitochondria. To generate detectable TPEF signals from NADH molecules in mitochondria, femtosecond laser pulses, instead of picosecond pulses as used in the current work, are needed. Whether the femtosecond pulses will cause an excess amount of photo-toxicity and perturbation to live cells that would alter the dynamics of organelles needs further investigation. This study is beyond the scope of this current work and will be performed in the future. 


\section{Materials and Methods}

Coherent anti-Stokes Raman scattering and multiphoton fluorescence microscopy

Our CARS microscope was constructed based on an upright microscope frame (BX51, Olympus). A dual-output laser system (Chameleon Discovery, Coherent Inc.) was implemented to generate a fixed laser beam at $1040 \mathrm{~nm}$, used as the pump beam, and a tunable laser beam (from $660 \mathrm{~nm}$ to $1300 \mathrm{~nm}$ ), used as the Stokes beam. The pulse width of both laser beams was $100 \mathrm{fs}$. To reduce peak power and photo-toxicity, we used a pair of SF57 glass rods (152.4 mm length for each, Lattice Electro-Optics) to chirp the pulses to $\sim 1$ ps for pump and $\sim 2$ ps for Stokes beams after combining with a dichroic mirror (see Figure S1). The power of the pump and Stokes beams at the sample was $14 \mathrm{~mW}$ and $10 \mathrm{~mW}$, respectively. The CARS signal was acquired by a photomultiplier tube (PMT) (H7422-40, Hamamatsu) placed in the transmission direction with a bandpass filter (650/13 nm, FF01-650/13/25, Semrock) to reject the excitation beams and autofluorescence signals from the sample. Multiphoton fluorescence signals from BODIPY-labeled lipids were collected by an epi-direction PMT (H7422-40, Hamamatsu) with a collection of dichroic mirrors and filters (785 \pm BW nm, Di02-R785-25×36, Semrock). Two pre-amplifiers (PMT-4V3, Advanced Research Instruments Corp.) were used to amplify the signals from PMT before the data acquisition system (PCle-6351, National Instruments). The water immersion objective lens used in this work (LUMPLFLN, Olympus) had a magnification of $40 \mathrm{X}$ and a numerical aperture of 0.8 .

\section{Image acquisition and the analysis of LD dynamics}

The lab-written image acquisition software was based on LabView. By setting the galvo mirror step voltage as $0.003 \mathrm{~V}$, a $400 \times 400$-pixel image covered a field-of-view (FOV) area of $85 \times 85 \mu \mathrm{m}^{2}$. CARS images for LD dynamic analysis containing $400 \times 400$ pixels were collected with a pixel dwell time of $10 \mu \mathrm{s}$, corresponding to $1.6 \mathrm{~s}$ per image. Considering extra edge-pixels on each line which will be removed after the acquisition and the galvo mirror returning process, the imaging speed is $2.2 \mathrm{~s} /$ frame. A total of 100 frames were acquired continuously for each FOV to record the LD dynamics. For all the LD dynamic analyses in this work, typically 5-9 FOVs were analyzed for each condition to provide statistical quantification. The image stacks were examined immediately after image collection. The inferior image stacks containing whole-cell movement, stage drift, focus drift, or other issues were excluded from the analysis.

For each FOV, the image stack was saved as a .txt file $(40,000 \times 400$ pixels), converted to a $100-$ frame image stack, and analyzed by a Particle Tracker ImageJ Plugin (44) using the following values for all parameters: radius $=0$, cutoff $=3$, percentile $=0.5-2$ (depends on the LD amount in the image), link range $=1$, displacement $=5$. The percentile was chosen to ensure the oversampling of LDs in images, i.e. picking up more particles than the LDs. The false trajectories from non-LD detections will be eliminated after applying a 20 -frame filter. All trajectories after particle linking were saved in a .txt file for quantitative analysis. Software written in MATLAB was used to quantify the trajectories. The maximum displacement $(\operatorname{maxd})$ was defined as the maximum displacement of the LD movement during the time of image acquisition. The LD speed equaled the total trajectory length divided by the total time. The directional factor (DF) was defined as the total trajectory length divided by the maxd, which was used to determine the directionality of the trajectory. The trajectory shown in Figure $1 \mathrm{C}$ was generated using the ImageJ Particle Tracker Plugin (the left panel: visualize all trajectories $\rightarrow$ double click the selected trajectory $\rightarrow$ save as .png) and plotted using Origin 2020 (the right panel).

The dynamic signature of the cells under a specific condition was plotted in 3D using MATLAB, with the projections on three planes. The contour density graph of the dynamic signature in each plane was plotted using MATLAB with a modified jet color scale by replacing the lowest blue color with white. The plot ranges for different graphs were scaled with maximum values detected throughout the study (maxd: -0.5 to 8.5 , speed: -0.024 to 0.24 , length: -1 to $25, D F:-1$ to 25 ). The 
level step for the contour plots was 0.00004 . Trajectory gating and subpopulation 2D plots were performed using MATLAB. The fitting of LD trajectories in the DF-maxd domain using a reciprocal function (see Figure 1J) was carried out by Origin 2020 and plotted by MATLAB.

Histograms of the LD dynamics on each axis were created using the ImageJ histogram function with 50 bins and fixed min and max values ( $\min =0, \max =8.5$ for maxd, 0.24 for speed, 25 for $D F$ and length). The histograms were normalized by dividing the total number of trajectories after 20 frame-filtering, then multiplied by $100 \%$. The histograms were plotted and fit using a single or dual lognormal distribution function in Origin 2020.

The single-lognormal function is defined as

$y=y_{0}+\frac{A}{\sqrt{2 \pi} w x} \exp \left[-\frac{\left(\ln \frac{x}{X_{c}}\right)^{2}}{2 w^{2}}\right]$

and $\sigma=\exp \left[\ln \left(X_{c}\right)+\frac{1}{2} w^{2}\right] \cdot \sqrt{\exp \left(w^{2}\right)-1}$

The dual-lognormal function requires self-definition and is defined as

$y=y_{0}+\frac{A_{1}}{\sqrt{2 \pi} w_{1} x} \exp \left[-\frac{\left(\ln \frac{x}{X_{c 1}}\right)^{2}}{2 w_{1}^{2}}\right]+\frac{A_{2}}{\sqrt{2 \pi} w_{2} x} \exp \left[-\frac{\left(\ln \frac{x}{X_{c 2}}\right)^{2}}{2 w_{2}^{2}}\right]$.

During the fitting, the lower bounds of all parameters were set to 0 . The $y_{0}$ intercept was also set to 0 . The $X c$ and $\sigma$ values were derived from the fitting for comparison. Dual lognormal distribution was only used to fit the speed histogram and gave the $A_{1}$ and $A_{2}$ values roughly equal to the fraction of LDs in each population.

To quantify the significance of changes in LD dynamics, we performed student's t-tests for LD trajectory information from different conditions. We averaged the measured parameters, e.g. maxd, speed, length, and DF, for each image stack. Then 5-9 FOVs (n values) were analyzed for each condition to create statistical tables of the parameters. Levels of significance were determined by calculating the $p$-values comparing the parameter tables from different groups (usually the treatment group v.s. the control group). The asterisks and n.s. have the following meanings: n.s., $p>0.05 ;{ }^{*}, 0.01<p<0.05 ;{ }^{* *}, 0.001<p<0.01 ;{ }^{* *}, p<0.001$.

For all CARS images, a single frame from the .txt image stack file was converted to a .png file for display. For the intensity thresholding, a single image frame was selected for each FOV. We first applied a Gaussian blur function (radius=15) to each image for 10 times (Figure S15B). Then we divided the original image by the Gaussian-blurred image to generate the LD-enhanced image (Figure S15C). This process was used to reduce the impact of background and cell intensity inhomogeneity on the LD particle analysis. From the processed image, we carried out intensity thresholding and used the Particle Analyzer Plugin in ImageJ, selecting particle size $<100$ pixels to crop the LD areas (Figure S15F,G). From the original image, we performed intensity thresholding and used particle analyzer to crop the cell area by defining particle sizes $>1000$ pixels (Figure S15D,E). Finally, the LD area percentage was calculated as the total LD area divided by the total cell area, multiplied by $100 \%$. 


\section{Temperature control}

Except where specifically mentioned, all LD dynamics measurements were performed at $37^{\circ} \mathrm{C}$. The temperature was controlled by a culture dish heating plate (DH-35iL, culture dish incubator, Warner Instruments). To maintain the temperature and reduce media evaporation, we used wet tissue paper to cover the open space between the heating plate and the objective lens. Cells removed from the incubator were immediately placed on the heating plate, unless otherwise mentioned. For hypothermia exposure, the heating plate was turned off and allowed for 5-10 min temperature stabilization to room temperature at $24^{\circ} \mathrm{C}$. The exposure at a given temperature lasted for different periods for different experiments. For reheating, the heating plate was turned back on and 5-10 min was allowed for temperature stabilization at $37^{\circ} \mathrm{C}$. Image collection was started immediately after temperature stabilization.

\section{Cell culture}

MIAPaCa-2, MDA-MB-231, and H358 cells were obtained from ATCC. The cells were normally cultured in DMEM $((+) 4.5 \mathrm{~g} / \mathrm{L}$ glucose, (+)glutamine, (-)sodium pyruvate) mixed with $10 \%$ FBS and $1 \%$ penicillin-streptomycin $(10,000 \mathrm{U} / \mathrm{mL})$. For starvation, glucose-free DMEM was used to eliminate the glucose supply in the medium. The DMEM or glucose-free DMEM with or without FBS created four culture conditions used in the starvation studies, all with $1 \%$ antibiotics present. The cells were first seeded to a $\sim 15-20 \%$ confluency followed by $24 \mathrm{hr}$ growth before all the studies or treatments.

\section{Chemical treatment of living cells}

Staurosporine (STS) was obtained from Sigma Aldrich. The Stock solution of STS in dimethyl sulfoxide (DMSO) has a concentration of $1 \mathrm{mM}$. MIAPaCa-2 cells were treated with STS to reach $500 \mathrm{nM}$ concentrations. Cells were first measured at $37^{\circ} \mathrm{C}$, without any treatment, as the control. Then STS was added to the media to reach the final concentration. CARS image stacks were collected immediately after the treatment, up to one hour. Then the sample was returned to and maintained in the incubator for the later $3 \mathrm{hr}$ and $11 \mathrm{hr}$ measurements. Similar procedures were used for the $1 \mu \mathrm{M}$ STS treatment.

AICAR treatment of MIAPaCa-2 cells were performed at $1 \mathrm{mM}$ concentration in normal $((+)$ glucose, (+)FBS) and starved media ((-)glucose, (-)FBS). The treatment time was $24 \mathrm{hr}$ for each condition. Chemicals including ranolazine $(80 \mu \mathrm{M})$, etomoxir $(20 \mu \mathrm{M})$, tunicamycin $(1 \mu \mathrm{g} / \mathrm{mL})$, $\mathrm{C} 75(50 \mu \mathrm{M})$, and trimetazidine $(1 \mathrm{mM})$ were used to inhibit different fatty acid-related enzymes or creating ER stress. The concentrations of these chemicals were selected based on those used in the literature to ensure cell viability. All treatments were performed for $24 \mathrm{hr}$. All the chemicals mentioned above were purchased from Cayman Chemical Company (Ann Arbor, MI) and used immediately after arrival.

\section{Fluorescent labeling}

The BODIPY green (493/503) was purchase from Cayman Chemical Company. A stock solution of $2 \mathrm{mg} / \mathrm{mL}$ BODIPY in DMSO was prepared for labeling. Finial concentrations of BODIPY in MIAPaCa-2 cells were $2 \mu \mathrm{g} / \mathrm{mL}$ and $4 \mu \mathrm{g} / \mathrm{mL}$ for different treatments. CARS images and TPEF images were acquired simultaneously from the two channels on the microscope. The LD dynamics analysis was performed using the CARS images, similar to other cases.

ER-Tracker ${ }^{\mathrm{TM}}$ Green was purchased from ThermoFisher. The stock solution had a concentration of $1 \mathrm{mM}$ in DMSO. The final concentration of ER-Tracker ${ }^{\mathrm{TM}}$ Green for ER labeling was $1 \mu \mathrm{M}$ and the cells were incubated with the dye for $30 \mathrm{~min}$ at $37^{\circ} \mathrm{C} / 5 \% \mathrm{CO}_{2}$ before imaging.

\section{Cell viability assay}

Thiazolyl Blue Tetrazolium Blue (M5655, Sigma Aldrich) (MTT) colorimetric assay was used to measure cell viability. Cells were first seeded in 96 -well plates and incubated overnight. Then the 
inhibitor treatments were performed using AICAR at different concentrations in the normal culture medium. A volume of $100 \mu \mathrm{L}$ medium was used for each well. Treatment time was $24 \mathrm{hr}$. The MTT stock solution was prepared at $5 \mathrm{mg} / \mathrm{mL}$ in culture medium and was added at one-tenth volume to each well. After $3 \mathrm{hr}$ incubation with the MTT solution, the medium in the wells was replaced by $100 \mu \mathrm{L}$ of DMSO and shaken well before plate reading. The sampling size was $n=6$ for each group. The plate was read by a BD plate reader. The $570 \mathrm{~nm}$ absorption was measured for all plates while the $690 \mathrm{~nm}$ absorption was also measured as the reference.

\section{Acknowledgments}

The authors thank Aneesh Alex, Jang Hyuk Lee, and Jose Rico-Jimenez from the Center for Optical Molecular Imaging for the initial installation of laser and data acquisition/PC systems, and thank GlaxoSmithKline for the sponsored research support of the Center. We also thank Darold Spillman for lab management and information technology support. This research was financially supported in part by grants from the NIH (R01CA241618, R01EB023232) and the NSF (CBET 18-41539). Additional information can be found at http://biophotonics.illinois.edu.

\section{Declaration of Interests}

Stephen Boppart is a co-founder and consultant of LiveBx, Champaign, IL, which is licensing intellectual property from the University of Illinois at Urbana-Champaign to develop novel optical sources and label-free multimodal multiphoton imaging platforms for biological and medical applications. Chi Zhang declares no competing interests.

\section{Supplemental Information}

Supplementary figures and videos are available.

\section{References}

Ahlemeyer, B., Klumpp, S., and Krieglstein, J. (2002). Release of cytochrome c into the extracellular space contributes to neuronal apoptosis induced by staurosporine. Brain Res. 934, 107-116.

Baenke, F., Peck, B., Miess, H., and Schulze, A. (2013). Hooked on fat: the role of lipid synthesis in cancer metabolism and tumour development. Dis. Model. Mech. 6, 13531363.

Bailey, A.P., Koster, G., Guillermier, C., Hirst, E.M., MacRae, J.I., Lechene, C.P., Postle, A.D., and Gould, A.P. (2015). Antioxidant role for lipid droplets in a stem cell niche of Drosophila. Cell 163, 340-353.

Banerjee, A., Lang, J.-Y., Hung, M.-C., Sengupta, K., Banerjee, S.K., Baksi, K., and Banerjee, D.K. (2011). Unfolded protein response is required in nu/nu mice microvasculature for treating breast tumor with tunicamycin. J. Biol. Chem. 286, 29127-29138.

Belmokhtar, C.A., Hillion, J., and Segal-Bendirdjian, E. (2001). Staurosporine induces apoptosis through both caspase-dependent and caspase-independent mechanisms. Oncogene 20, 3354-3362. 
Biron-Shental, T., Schaiff, W.T., Ratajczak, C.K., Bildirici, I., Nelson, D.M., and Sadovsky, Y. (2007). Hypoxia regulates the expression of fatty acid-binding proteins in primary term human trophoblasts. Am. J. Obstet. Gynecol. 197, 516. e511-516. e516.

Bosma, M., Dapito, D.H., Drosatos-Tampakaki, Z., Huiping-Son, N., Huang, L.-S., Kersten, S., Drosatos, K., and Goldberg, I.J. (2014). Sequestration of fatty acids in triglycerides prevents endoplasmic reticulum stress in an in vitro model of cardiomyocyte lipotoxicity. BBA-Mol. Cell Bio. L 1841, 1648-1655.

Bradley, J., Pope, I., Masia, F., Sanusi, R., Langbein, W., Swann, K., and Borri, P. (2016). Quantitative imaging of lipids in live mouse oocytes and early embryos using CARS microscopy. Development 143, 2238-2247.

Camp Jr, C.H., Lee, Y.J., Heddleston, J.M., Hartshorn, C.M., Walker, A.R.H., Rich, J.N., Lathia, J.D., and Cicerone, M.T. (2014). High-speed coherent Raman fingerprint imaging of biological tissues. Nat. Photonics 8, 627.

Cheng, J.-X., Volkmer, A., and Xie, X.S. (2002). Theoretical and experimental characterization of coherent anti-Stokes Raman scattering microscopy. J. Opt. Soc. Am. B 19, 13631375.

Cheng, J.-X., and Xie, X.S. (2015). Vibrational spectroscopic imaging of living systems: An emerging platform for biology and medicine. Science 350, aaa8870.

Cotte, A.K., Aires, V., Fredon, M., Limagne, E., Derangère, V., Thibaudin, M., Humblin, E., Scagliarini, A., de Barros, J.-P.P., and Hillon, P. (2018). Lysophosphatidylcholine acyltransferase 2-mediated lipid droplet production supports colorectal cancer chemoresistance. Nat. Commun. 9, 1-16.

Di Napoli, C., Pope, I., Masia, F., Watson, P., Langbein, W., and Borri, P. (2014). Hyperspectral and differential CARS microscopy for quantitative chemical imaging in human adipocytes. Biomed. Opt. Express 5, 1378-1390.

Evans, C.L., Potma, E.O., Puoris' haag, M., Côté, D., Lin, C.P., and Xie, X.S. (2005). Chemical imaging of tissue in vivo with video-rate coherent anti-Stokes Raman scattering microscopy. Proc. Natl. Acad. Sci. U. S. A. 102, 16807-16812.

Freudiger, C.W., Min, W., Saar, B.G., Lu, S., Holtom, G.R., He, C., Tsai, J.C., Kang, J.X., and Xie, X.S. (2008). Label-free biomedical imaging with high sensitivity by stimulated Raman scattering microscopy. Science 322, 1857-1861.

Fu, D. (2017). Quantitative chemical imaging with stimulated Raman scattering microscopy. Curr. Opin. Chem. Biol. 39, 24-31.

Fu, D., Lu, F.-K., Zhang, X., Freudiger, C., Pernik, D.R., Holtom, G., and Xie, X.S. (2012). Quantitative chemical imaging with multiplex stimulated Raman scattering microscopy. J. Am. Chem. Soc. 134, 3623-3626.

Hardie, D.G. (2016). Regulation of AMP-activated protein kinase by natural and synthetic activators. Acta Pharm. Sin. B 6, 1-19.

Hardie, D.G., Ross, F.A., and Hawley, S.A. (2012). AMPK: a nutrient and energy sensor that maintains energy homeostasis. Nat. Rev. Mol. Cell Biol. 13, 251-262.

He, R., Xu, Y., Zhang, L., Ma, S., Wang, X., Ye, D., and Ji, M. (2017). Dual-phase stimulated Raman scattering microscopy for real-time two-color imaging. Optica 4, 44-47.

Herms, A., Bosch, M., Reddy, B.J., Schieber, N.L., Fajardo, A., Rupérez, C., Fernández-Vidal, A., Ferguson, C., Rentero, C., and Tebar, F. (2015). AMPK activation promotes lipid droplet dispersion on detyrosinated microtubules to increase mitochondrial fatty acid oxidation. Nat. Commun. 6, 1-14.

Jarc, E., Kump, A., Malavašič, P., Eichmann, T.O., Zimmermann, R., and Petan, T. (2018). Lipid droplets induced by secreted phospholipase A2 and unsaturated fatty acids protect breast cancer cells from nutrient and lipotoxic stress. BBA-Mol. Cell Bio. L 1863, 247265. 
Kantor, P.F., Lucien, A., Kozak, R., and Lopaschuk, G.D. (2000). The antianginal drug trimetazidine shifts cardiac energy metabolism from fatty acid oxidation to glucose oxidation by inhibiting mitochondrial long-chain 3-ketoacyl coenzyme A thiolase. Circ. Res. 86, 580-588.

Kim, E.-K., Miller, I., Aja, S., Landree, L.E., Pinn, M., McFadden, J., Kuhajda, F.P., Moran, T.H., and Ronnett, G.V. (2004). C75, a fatty acid synthase inhibitor, reduces food intake via hypothalamic AMP-activated protein kinase. J. Biol. Chem. 279, 19970-19976.

Kim, J., Yang, G., Kim, Y., Kim, J., and Ha, J. (2016). AMPK activators: mechanisms of action and physiological activities. Exp. Mol. Med. 48, e224-e224.

Koizume, S., and Miyagi, Y. (2016). Lipid droplets: a key cellular organelle associated with cancer cell survival under normoxia and hypoxia. Int. J. Mol. Sci. 17, 1430.

Li, J., Condello, S., Thomes-Pepin, J., Ma, X., Xia, Y., Hurley, T.D., Matei, D., and Cheng, J.-X. (2017). Lipid desaturation is a metabolic marker and therapeutic target of ovarian cancer stem cells. Cell Stem Cell 20, 303-314. e305.

Liao, C.-S., Slipchenko, M.N., Wang, P., Li, J., Lee, S.-Y., Oglesbee, R.A., and Cheng, J.-X. (2015). Microsecond scale vibrational spectroscopic imaging by multiplex stimulated Raman scattering microscopy. Light Sci. Appl 4, e265-e265.

Liu, Q., Luo, Q., Halim, A., and Song, G. (2017). Targeting lipid metabolism of cancer cells: a promising therapeutic strategy for cancer. Cancer Lett. 401, 39-45.

Lu, F.-K., Basu, S., Igras, V., Hoang, M.P., Ji, M., Fu, D., Holtom, G.R., Neel, V.A., Freudiger, C.W., and Fisher, D.E. (2015). Label-free DNA imaging in vivo with stimulated Raman scattering microscopy. Proc. Natl. Acad. Sci. U. S. A. 112, 11624-11629.

Min, W., Freudiger, C.W., Lu, S., and Xie, X.S. (2011). Coherent nonlinear optical imaging: beyond fluorescence microscopy. Annu. Rev. Phys. Chem. 62, 507-530.

Mirkes, P.E., and Little, S.A. (2000). Cytochrome c release from mitochondria of early postimplantation murine embryos exposed to 4-hydroperoxycyclophosphamide, heat shock, and staurosporine. Toxicol. Appl. Pharmacol. 162, 197-206.

Mitra, R., Le, T.T., Gorjala, P., and Goodman Jr, O.B. (2017). Positive regulation of prostate cancer cell growth by lipid droplet forming and processing enzymes DGAT1 and ABHD5. BMC Cancer 17, 631.

Morjani, H., Aouali, N., Belhoussine, R., Veldman, R.J., Levade, T., and Manfait, M. (2001). Elevation of glucosylceramide in multidrug-resistant cancer cells and accumulation in cytoplasmic droplets. Int. J. Cancer 94, 157-165.

Nan, X., Potma, E.O., and Xie, X.S. (2006). Nonperturbative chemical imaging of organelle transport in living cells with coherent anti-stokes Raman scattering microscopy. Biophys. J. 91, 728-735.

Ozeki, Y., Umemura, W., Otsuka, Y., Satoh, S., Hashimoto, H., Sumimura, K., Nishizawa, N., Fukui, K., and Itoh, K. (2012). High-speed molecular spectral imaging of tissue with stimulated Raman scattering. Nat. Photonics 6, 845-851.

Palonpon, A.F., Sodeoka, M., and Fujita, K. (2013). Molecular imaging of live cells by Raman microscopy. Curr. Opin. Chem. Biol. 17, 708-715.

Pleitez, M.A., Khan, A.A., Soldà, A., Chmyrov, A., Reber, J., Gasparin, F., Seeger, M.R., Schätz, B., Herzig, S., and Scheideler, M. (2020). Label-free metabolic imaging by midinfrared optoacoustic microscopy in living cells. Nat. Biotechnol. 38, 293-296.

Potma, E.O., Jones, D.J., Cheng, J.-X., Xie, X.S., and Ye, J. (2002). High-sensitivity coherent anti-Stokes Raman scattering microscopy with two tightly synchronized picosecond lasers. Opt. Lett. 27, 1168-1170.

Reaven, G.M., Chang, H., and Hoffman, B.B. (1988). Additive hypoglycemic effects of drugs that modify free-fatty acid metabolism by different mechanisms in rats with streptozocininduced diabetes. Diabetes 37, 28-32. 
Rinia, H.A., Burger, K.N., Bonn, M., and Müller, M. (2008). Quantitative label-free imaging of lipid composition and packing of individual cellular lipid droplets using multiplex CARS microscopy. Biophys. J. 95, 4908-4914.

Sabbah, H.N., Chandler, M.P., Mishima, T., Suzuki, G., Chaudhry, P., Nass, O., Biesiadecki, B.J., Blackburn, B., Wolff, A., and Stanley, W.C. (2002). Ranolazine, a partial fatty acid oxidation ( $\mathrm{pFOX}$ ) inhibitor, improves left ventricular function in dogs with chronic heart failure. J. Card. Fail. 8, 416-422.

SALT, I.P., JOHNSON, G., ASHCROFT, S.J., and HARDIE, D.G. (1998). AMP-activated protein kinase is activated by low glucose in cell lines derived from pancreatic $\beta$ cells, and may regulate insulin release. Biochem. J. 335, 533-539.

Sbalzarini, I.F., and Koumoutsakos, P. (2005). Feature point tracking and trajectory analysis for video imaging in cell biology. J. Struct. Biol. 151, 182-195.

Slipchenko, M.N., Le, T.T., Chen, H., and Cheng, J.-X. (2009). High-speed vibrational imaging and spectral analysis of lipid bodies by compound Raman microscopy. J. Phys. Chem. B 113, 7681-7686.

Walther, T.C., and Farese Jr, R.V. (2012). Lipid droplets and cellular lipid metabolism. Annu. Rev. Biochem. 81, 687-714.

Wang, P., Li, J., Wang, P., Hu, C.R., Zhang, D., Sturek, M., and Cheng, J.X. (2013). Label-free quantitative imaging of cholesterol in intact tissues by hyperspectral stimulated raman scattering microscopy. Angew. Chem. Int. Ed. 52, $13042-13046$.

Welte, M.A. (2015). Expanding roles for lipid droplets. Curr. Biol. 25, R470-R481.

Yue, S., Li, J., Lee, S.-Y., Lee, H.J., Shao, T., Song, B., Cheng, L., Masterson, T.A., Liu, X., and Ratliff, T.L. (2014). Cholesteryl ester accumulation induced by PTEN loss and PI3K/AKT activation underlies human prostate cancer aggressiveness. Cell Metab. 19, 393-406.

Yue, T.-L., Wang, C., Romanic, A.M., Kikly, K., Keller, P., DeWolf Jr, W.E., Hart, T.K., Thomas, H.C., Storer, B., and Gu, J.-L. (1998). Staurosporine-induced apoptosis in cardiomyocytes: a potential role of caspase-3. J. Mol. Cell. Cardiol. 30, 495-507.

Zhang, C., Li, J., Lan, L., and Cheng, J.-X. (2017). Quantification of lipid metabolism in living cells through the dynamics of lipid droplets measured by stimulated Raman scattering imaging. Anal. Chem. 89, 4502-4507.

Zhang, D., Li, C., Zhang, C., Slipchenko, M.N., Eakins, G., and Cheng, J.-X. (2016). Depthresolved mid-infrared photothermal imaging of living cells and organisms with submicrometer spatial resolution. Sci. Adv. 2, e1600521.

Zhang, D., Wang, P., Slipchenko, M.N., Ben-Amotz, D., Weiner, A.M., and Cheng, J.-X. (2013). Quantitative vibrational imaging by hyperspectral stimulated Raman scattering microscopy and multivariate curve resolution analysis. Anal. Chem. 85, 98-106.

Zumbusch, A., Holtom, G.R., and Xie, X.S. (1999). Three-dimensional vibrational imaging by coherent anti-Stokes Raman scattering. Phys. Rev. Lett. 82, 4142. 


\section{Figures and Figure Legends}

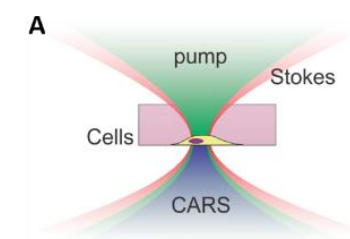

D

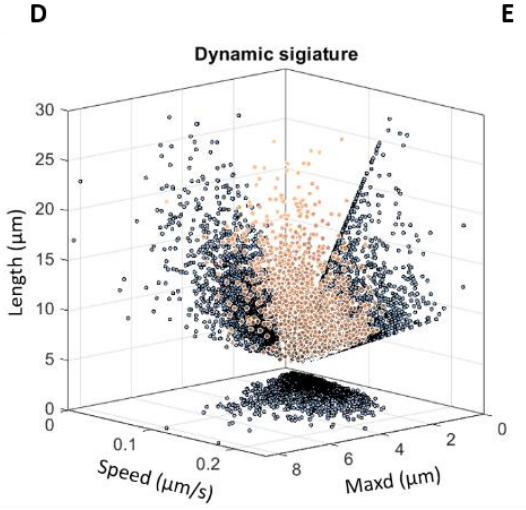

E

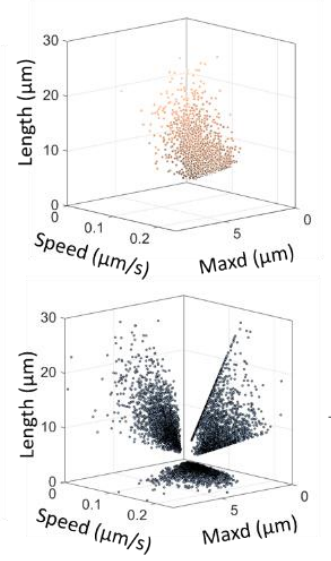

c

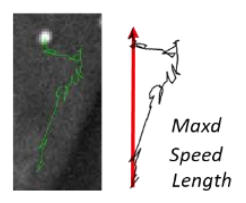

$\mathbf{F}$

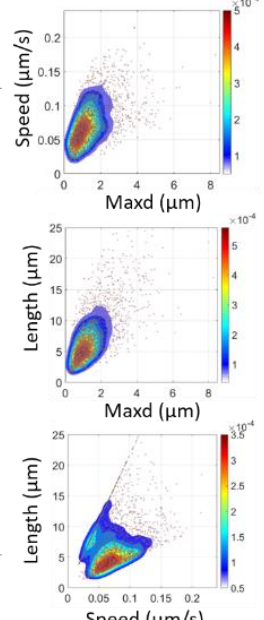

Speed $(\mu \mathrm{m} / \mathrm{s})$

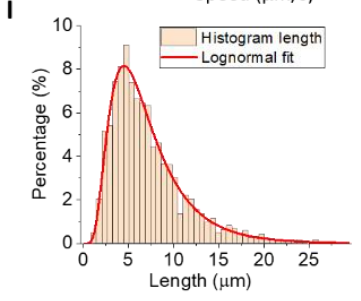

$\mathbf{L}$
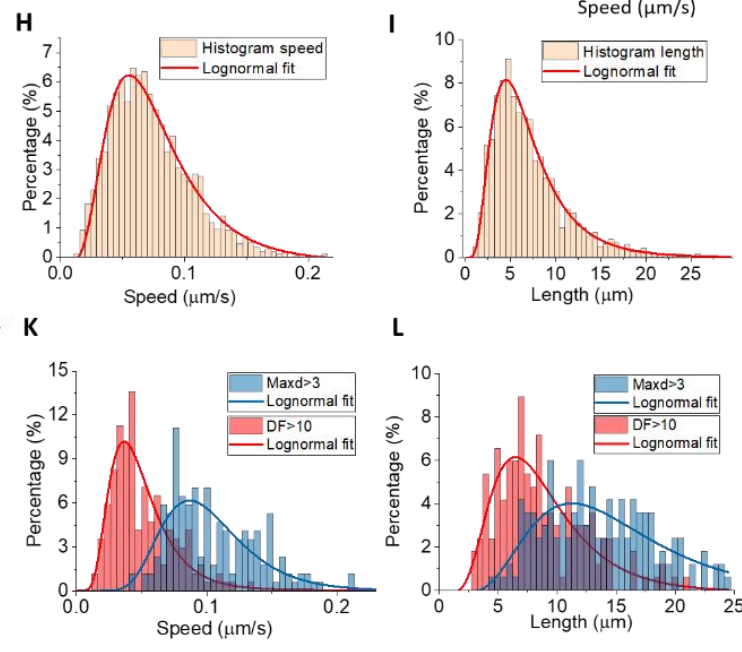

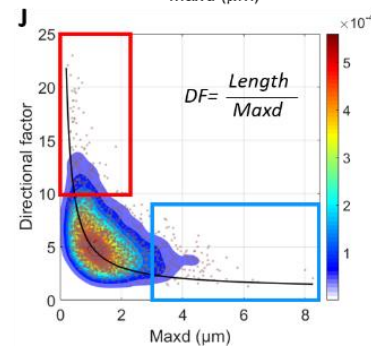

Figure 1. Coherent Raman scattering microscopy for quantitative LD dynamics study. (A) Schematic of CARS signal generation by living cells. (B) Time-lapse imaging of live cells using CARS. (C) An LD trajectory and the parameters derived directly from the trajectory, including the maximum displacement $(\operatorname{maxd})$, the average speed, and the total trajectory length. (D) Dynamic signature of MIAPaCa-2 cells plotted in the maxd, speed, and length 3D space. (E) The 3D plot of the MIAPaCa-2 dynamic signature without (upper panel) projections, and the projections onto the three 2D planes (bottom panel). (F) Density contour plot of the dynamic signature projected onto the speed-maxd, length-maxd, and length-speed planes. The dots are individual LD trajectories. (G)-(I) One-dimensional histograms of the MIAPaCa-2 dynamic signature projected onto the maxd, speed, and length axes, respectively, and the lognormal fitting of the histograms. (J) Density contour plot of the dynamic signature projected onto the DF-maxd plane. The dots are individual LD trajectories. The black curve is the fitting curve using the reciprocal function. (K) and (L) Histograms and lognormal fitting results of the gated trajectories in panel $(\mathrm{J})$. Red plots are trajectories with $D F>10$, while blue plots are trajectories with maxd $>3$. 
A

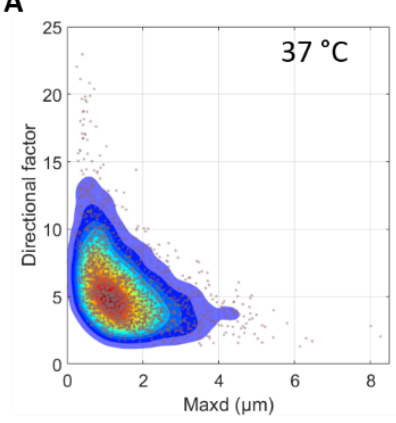

B

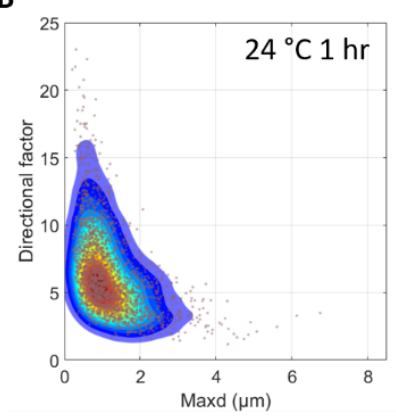

C

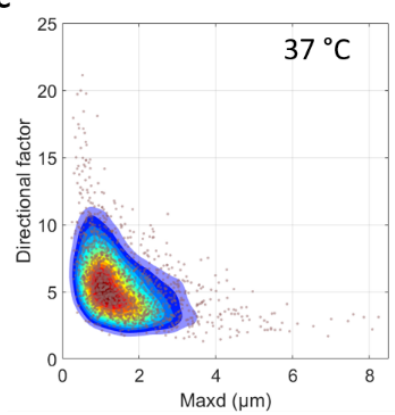

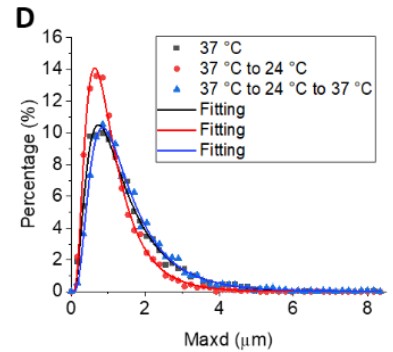

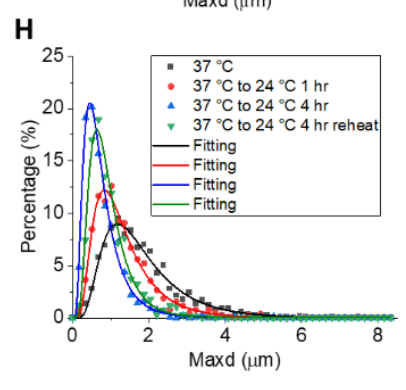

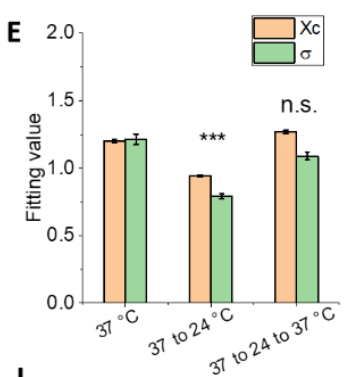

I

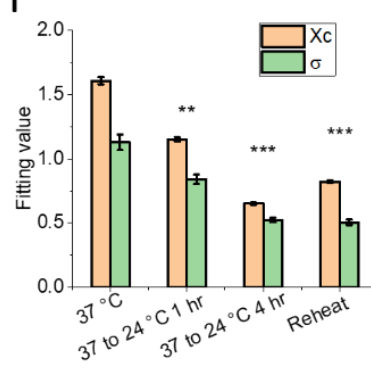

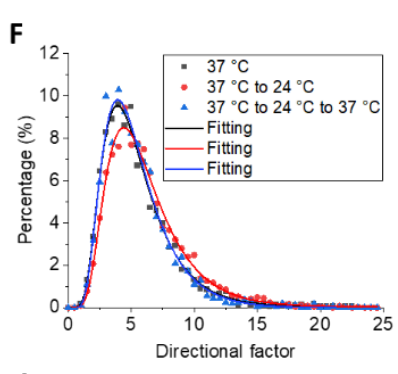

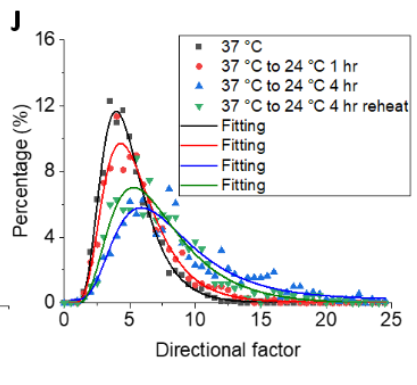

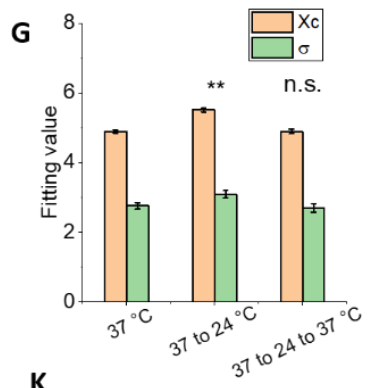

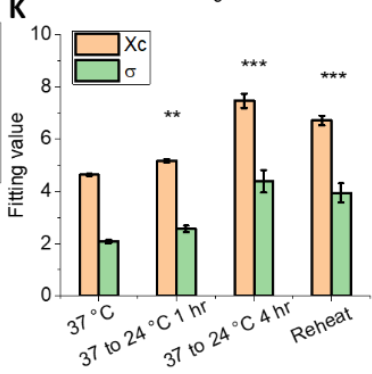

Figure 2. Detection and quantification of hypothermia-induced changes in LD dynamics. (A)-(C) Dynamic signatures of MIAPaCa-2 cells at $37^{\circ} \mathrm{C}$, hypothermia exposure to $24^{\circ} \mathrm{C}$ for $1 \mathrm{hr}$, and reheating of sample to $37^{\circ} \mathrm{C}$, respectively, projected onto the DF-maxd plane. (D) Histograms (dots) and the lognormal fitting of the histograms (solid curves) of MIAPaCa-2 cells in the maxd domain from the control $\left(37^{\circ} \mathrm{C}\right.$, black), $1 \mathrm{hr}$ hypothermia exposure $\left(24^{\circ} \mathrm{C}\right.$, red), and after reheating $\left(37^{\circ} \mathrm{C}\right.$, blue) conditions. (E) $X c$ and $\sigma$ derived from the lognormal fitting from each condition in panel (D). (F) Histograms (dots) and the lognormal fitting of the histograms (solid curves) of MIAPaCa-2 cells in the DF domain, from the three conditions. (G) $X c$ and $\sigma$ derived from the lognormal fitting from each condition in panel $(F)$. (H) Histograms (dots) and the lognormal fitting of the histograms (solid curves) of MIAPaCa-2 cells in the maxd domain from the control $\left(37^{\circ} \mathrm{C}\right.$, black), $1 \mathrm{hr}$ hypothermia exposure $\left(24^{\circ} \mathrm{C}\right.$, red $), 4 \mathrm{hr}$ hypothermia exposure $\left(24^{\circ} \mathrm{C}\right.$, blue), and after reheating $\left(37^{\circ} \mathrm{C}\right.$, green) conditions. (I) $X c$ and $\sigma$ derived from the lognormal fitting from each condition in panel $(\mathrm{H})$. $(\mathrm{J})$ Histograms (dots) and the lognormal fitting of the histograms (solid curves) of MIAPaCa-2 cells in the DF domain from the four conditions in panel $(\mathrm{H})$. (K) $X_{C}$ and $\sigma$ derived from the lognormal fitting from each condition in panel $(\mathrm{J})$. For the student's t-tests for the $1 \mathrm{hr}$ hypothermia exposure, $\mathrm{n}=9$, and the $4 \mathrm{hr}$ hypothermia exposure, $\mathrm{n}=7$. 
A

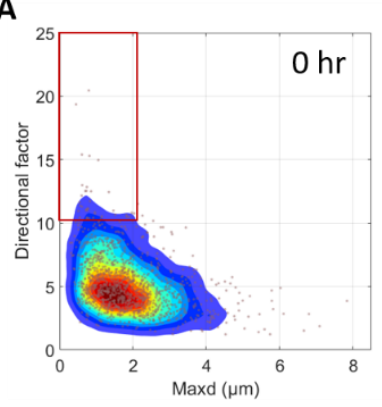

B

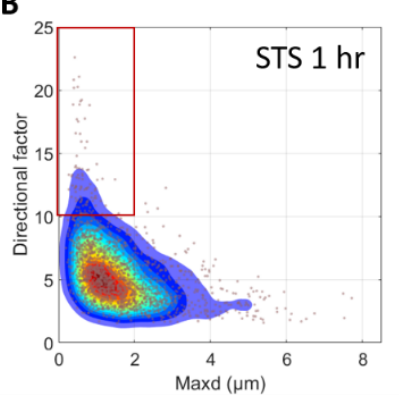

c

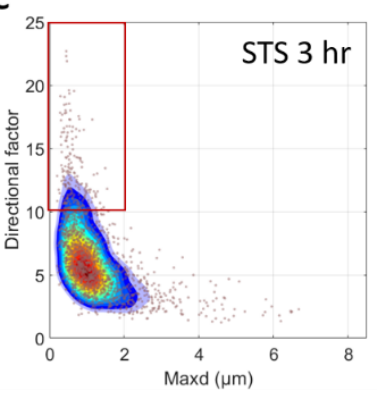

D

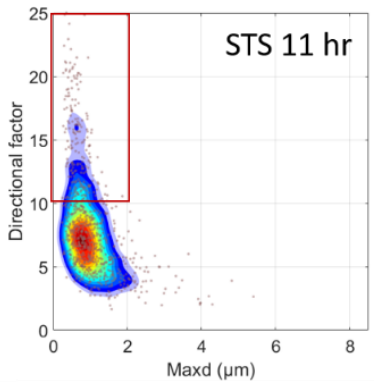

E

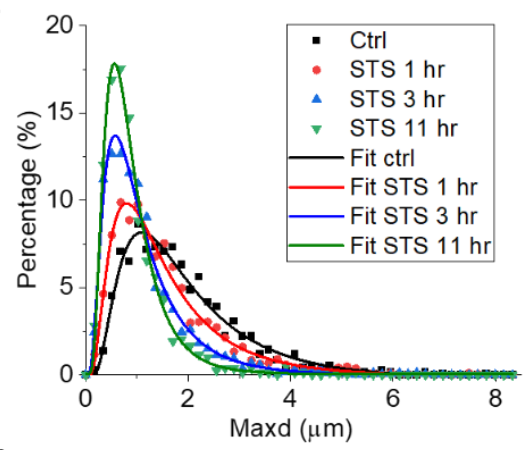

H

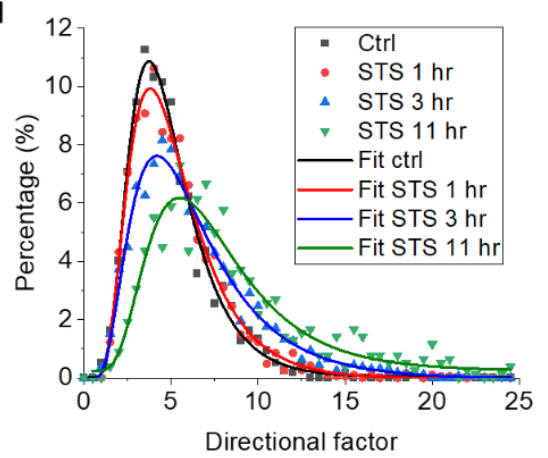

$\mathbf{F}$

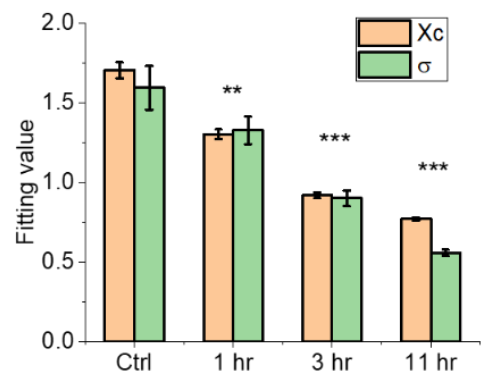

I

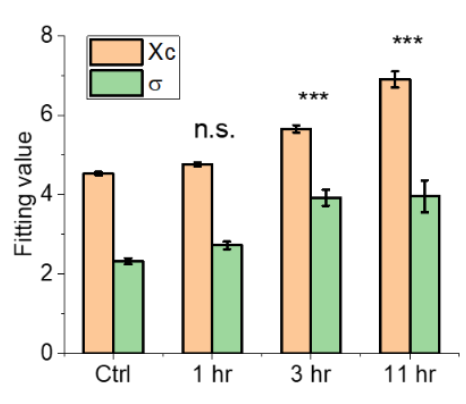

G

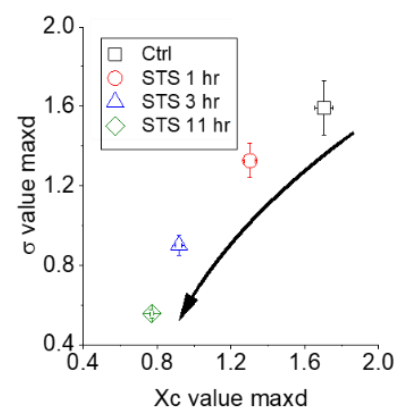

J

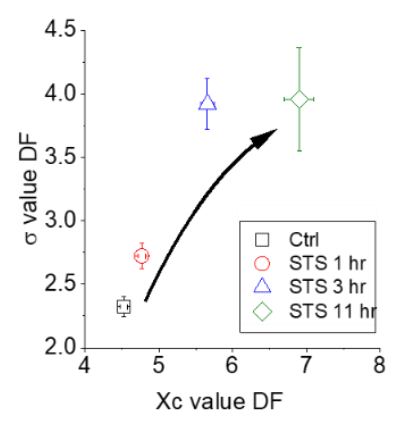

Figure 3. Changes in LD dynamics during apoptosis. (A)-(D) Dynamic signatures of MIAPaCa-2 control group, $0.05 \%$ STS treatment for $1 \mathrm{hr}, 3 \mathrm{hr}$, and $11 \mathrm{hr}$, respectively, projected onto the DF-maxd plane. The red rectangles are shown as reference marks. (E) Histograms (dots) and the lognormal fitting of the histograms (solid curves) of MIAPaCa-2 cells in the maxd domain from the control (black), $1 \mathrm{hr}$ STS treatment (red), $3 \mathrm{hr}$ STS treatment (blue), and $11 \mathrm{hr}$ STS treatment (green). (F) $X_{c}$ and $\sigma$ values derived from the lognormal fitting from each condition in panel (E). (G) Time-dependent STS treatment results plotted in the maxd $X_{C}-\sigma$ domain. The arrow indicates the change over STS treatment time. $(\mathrm{H})$ Histograms (dots) and the lognormal fitting of the histograms (solid curves) of MIAPaCa-2 cells in the DF domain from the four conditions. (I) $X_{C}$ and $\sigma$ values derived from the lognormal fitting of each condition in panel (G). (J) Timedependent STS treatment results plotted in the $D F X_{C-} \sigma$ domain. The arrow indicates the change over STS treatment time. For the student's t-tests, $n=6$. 

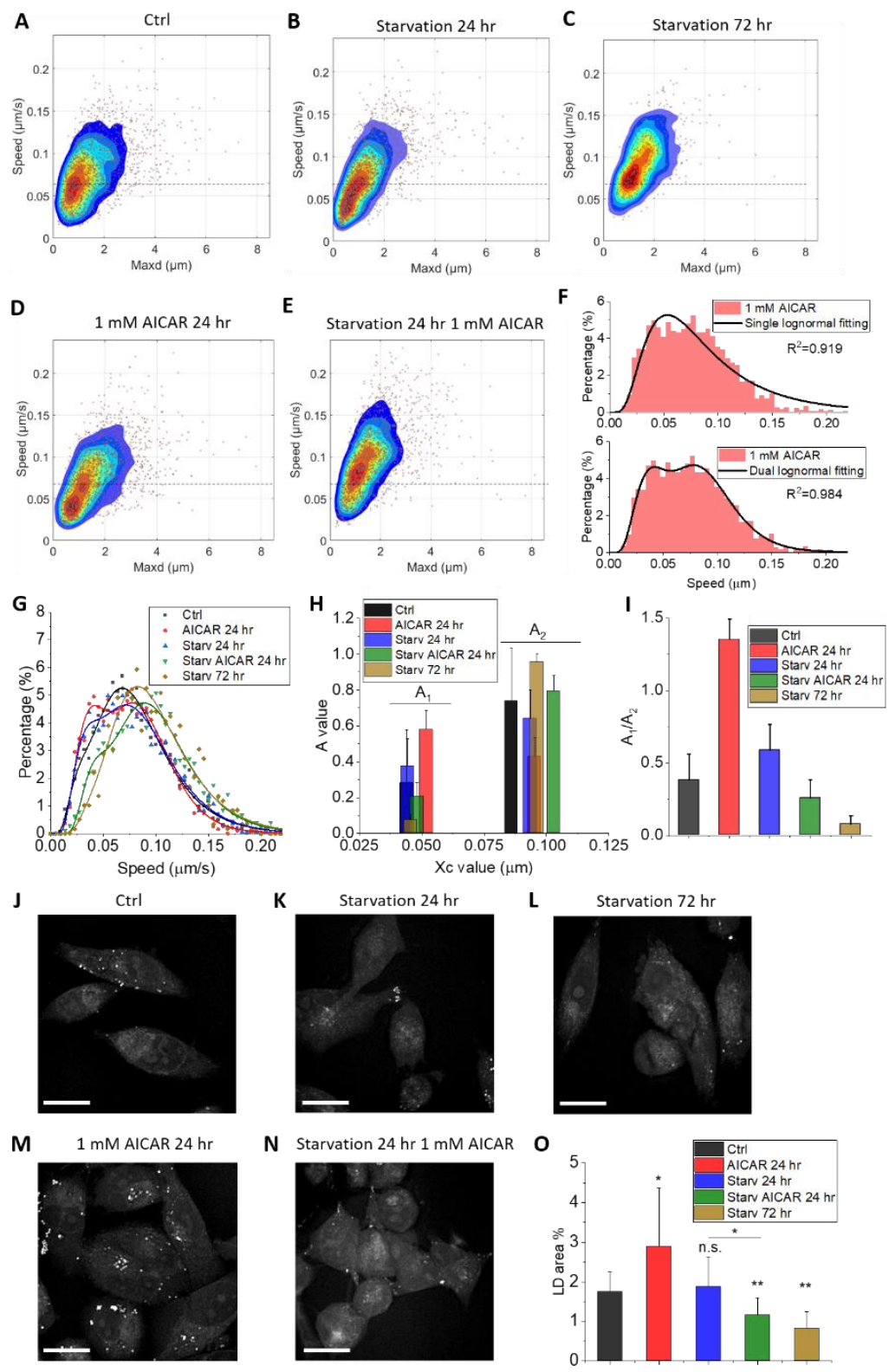

Figure 4. Changes in LD dynamics related to AMPK activation. (A)-(E) Dynamic signatures of the MIAPaCa-2 control, $24 \mathrm{hr}$ starvation ((-)glucose, (-)FBS), $72 \mathrm{hr}$ starvation, $1 \mathrm{mM}$ AICAR treatment in normal culture medium for $24 \mathrm{hr}$, and $1 \mathrm{mM}$ AICAR treatment in starvation condition for $24 \mathrm{hr}$, projected onto the speed-maxd plane, respectively. The dotted lines are shown as reference marks. (F) Comparing the fitting of speed histogram of MIAPaCa-2 cells using a single lognormal distribution function (upper panel) and a dual lognormal distribution function (lower panel). (G) Histograms (dots) and the dual-lognormal fitting of the histograms (solid curves) of the dynamics signatures in the speed domain for the five conditions in panels $(A-E)$. (H) XC and amplitude values reflect the median speed and the population fractions of the two subpopulations of LDs in MIAPaCa-2 cells in the five conditions. (I) The ratio of amplitudes $A_{1} / A_{2}$ for the five conditions. (J-N) Sample CARS images from the five conditions in greyscale. (O) Quantification of LD area percentage for the five conditions using the method described in Figure S15. For the student's t-tests, $\mathrm{n}=8$. 

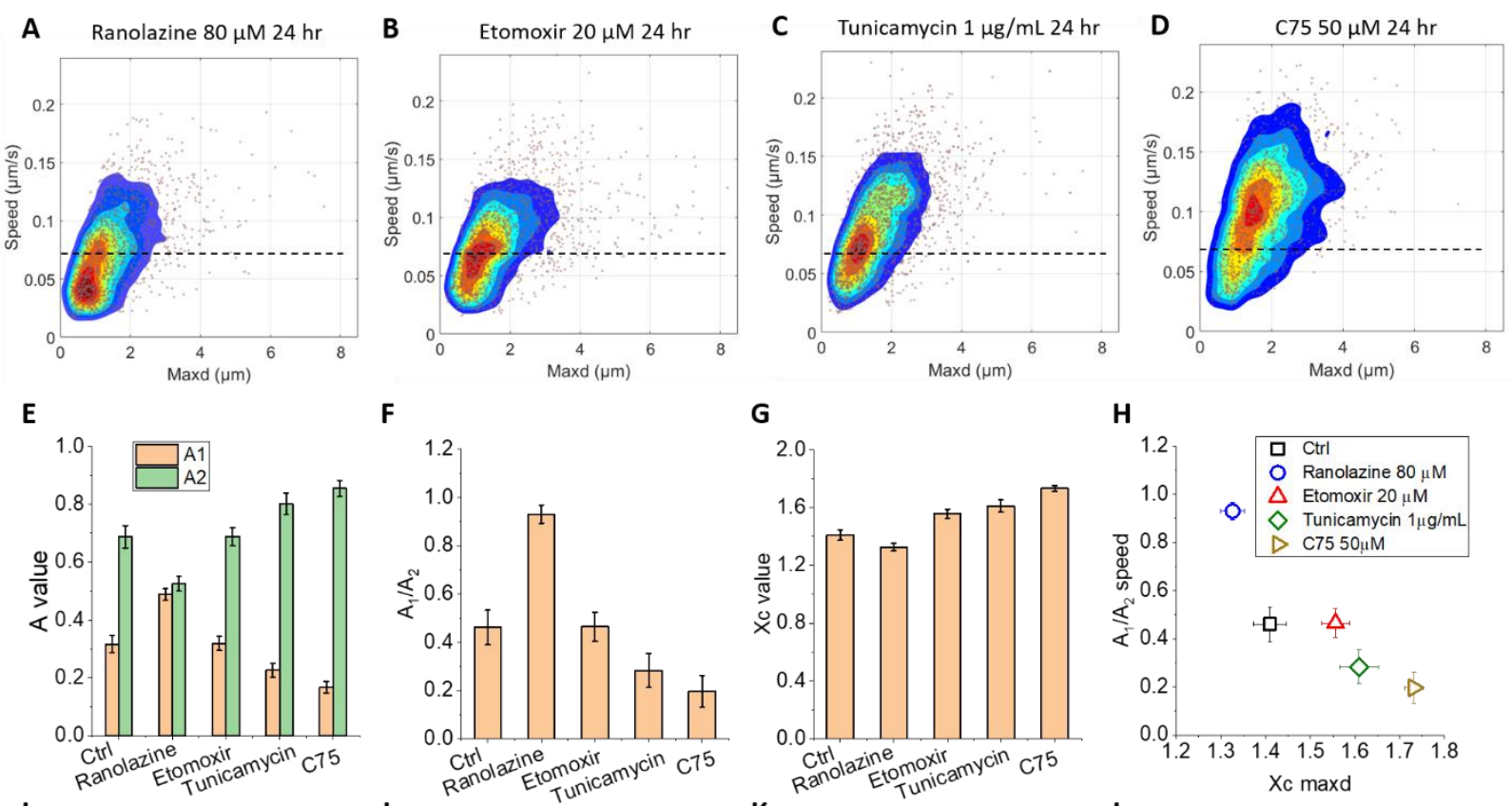

$\mathbf{F}$

G
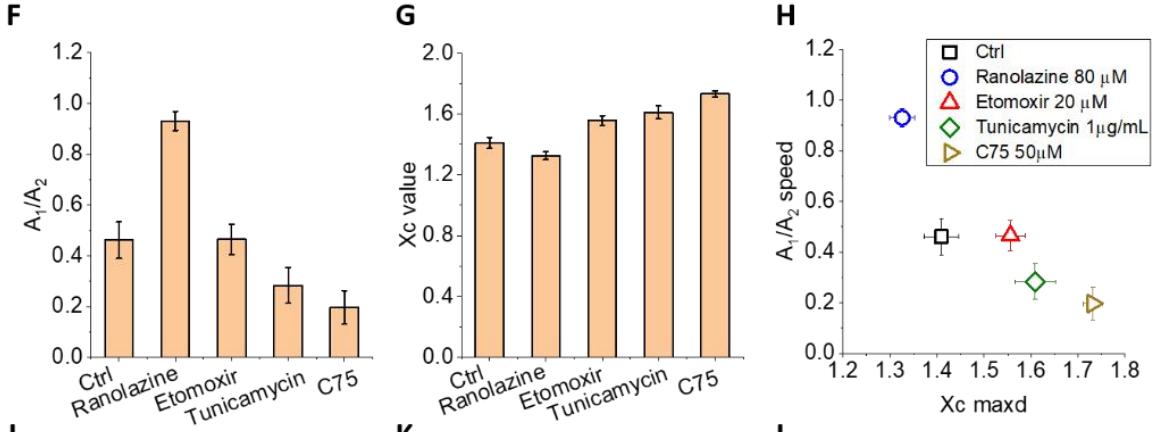

J
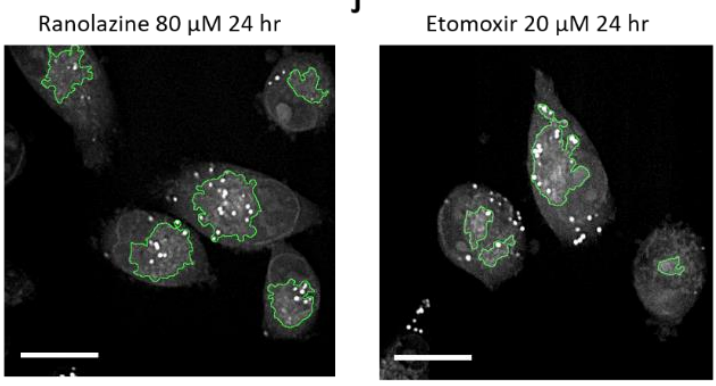

$\mathbf{K}$

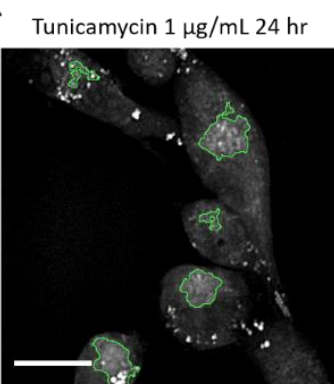

$\mathbf{L}$

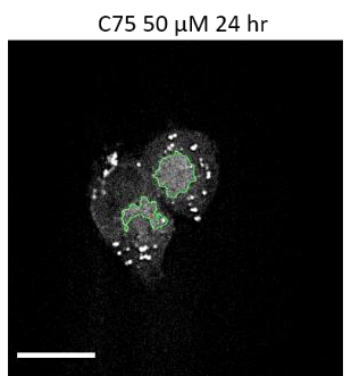

Figure 5. Changes in LD dynamics related to inhibition of lipid metabolic enzymes. (A)-(D) Dynamic signatures of MIAPaCa-2 treated by $80 \mu \mathrm{M}$ ranolazine, $20 \mu \mathrm{M}$ etomoxir, $1 \mu \mathrm{g} / \mathrm{mL}$ tunicamycin, and $50 \mu \mathrm{M} \mathrm{C75}$, projected onto the speed-maxd plane, respectively. The dotted lines are shown as reference marks. (E) Amplitudes $A_{1}$ and $A_{2}$ values, reflecting the fractions of two subpopulations of LDs in cells, derived from fitting speed histograms using dual-lognormal distribution. (F) The ratio of amplitudes $A_{1} / A_{2}$ for the five conditions. (G) The $X_{c}$ values obtained from fitting maxd histograms using single-lognormal distribution functions. $(\mathrm{H})$ The control group and four treatment groups plotted on the $A_{1} / A_{2}-$ maxd $X_{C}$ domain. (I-L) Sample images of MIAPaCa-2 cells from the four treatment groups. The green area plots the intensity-thresholdinghighlighted ER areas. The intensity thresholding method for ER analysis is illustrated in Figure S7. 


\section{Supplementary Information for}

\section{Dynamic signatures of lipid droplets as new markers to quantify cellular metabolic changes}

Chi Zhang ${ }^{1}$ and Stephen A. Boppart ${ }^{1,2,3,4,5}$

${ }^{1}$ Beckman Institute for Advanced Science and Technology

${ }^{2}$ Department of Bioengineering

${ }^{3}$ Department of Electrical and Computer Engineering

${ }^{4}$ Carle Illinois College of Medicine

${ }^{5}$ Cancer Center at Illinois

University of Illinois Urbana Champaign

*Stephen A. Boppart, boppart@illinois.edu 


\section{Supplementary Figures}
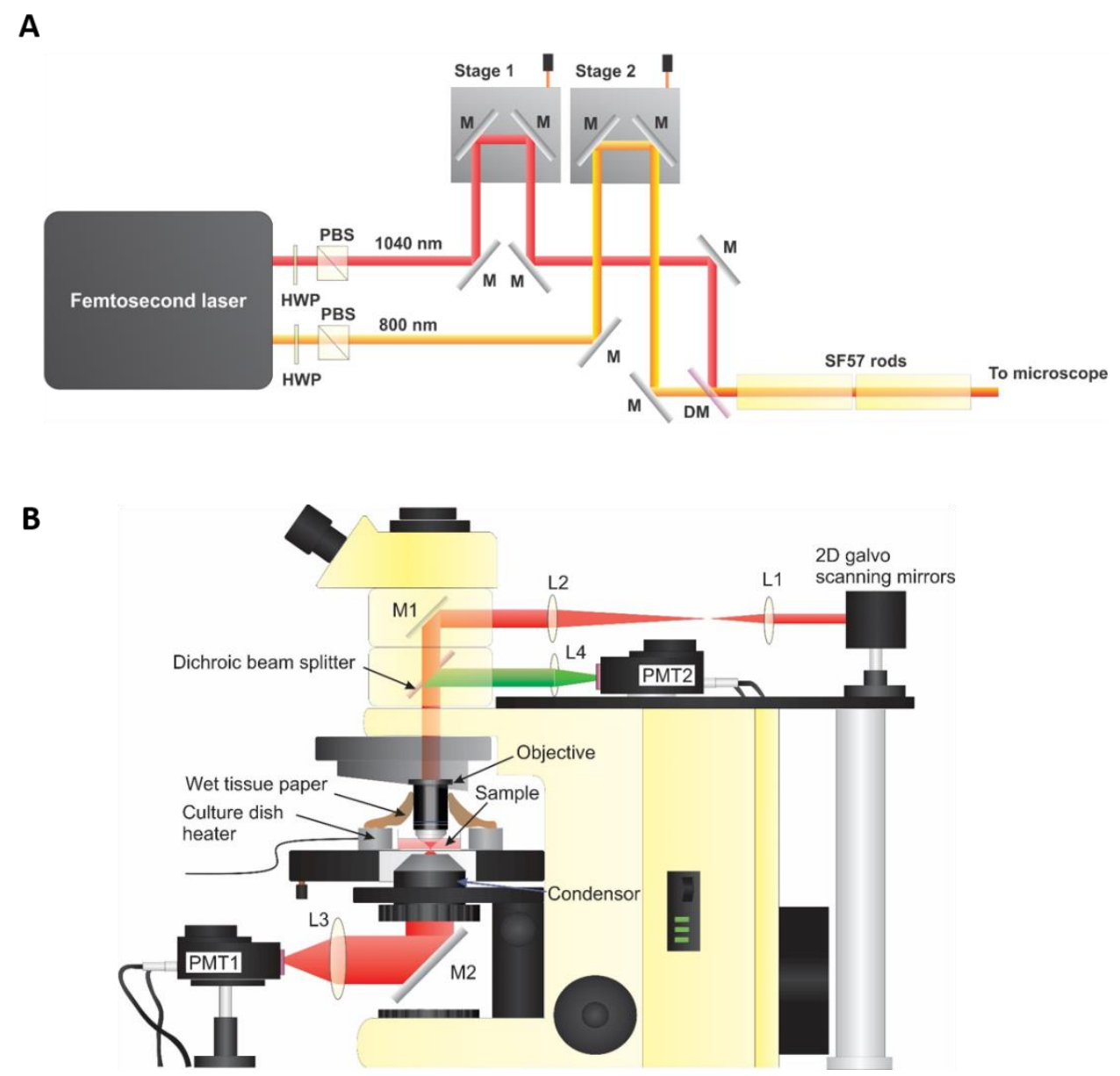

Figure S1. Experimental setup. (A) A schematic showing the coherent anti-Stokes Raman scattering optical paths before the laser-scanning microscope. M: mirrors; PBS: polarization beam splitter; HWP: half-wave-plate; DM: dichroic mirror. (B) A schematic showing the microscope setup used for hybrid-CARS-TPEF imaging. L: lens; M: mirror; PMT: photomultiplier tube. 
A

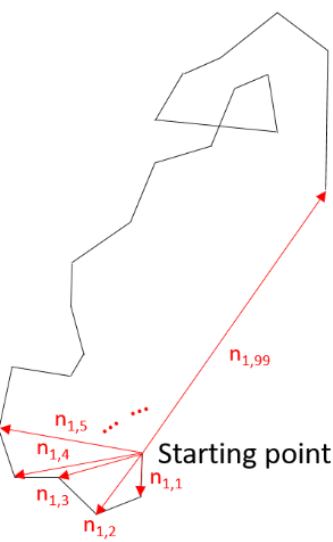

$\operatorname{maxd}_{1}=$ Maximum $\left(\mathrm{n}_{1,1}\right.$ to $\left.\mathrm{n}_{1,99}\right)$
B

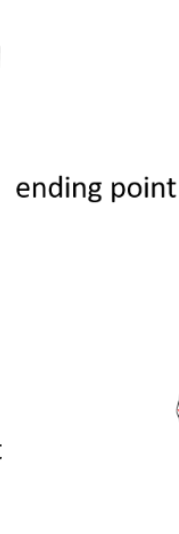

$\operatorname{maxd}_{2}=$ Maximum $\left(\mathrm{n}_{2,2}\right.$ to $\left.\mathrm{n}_{2,99}\right)$
C
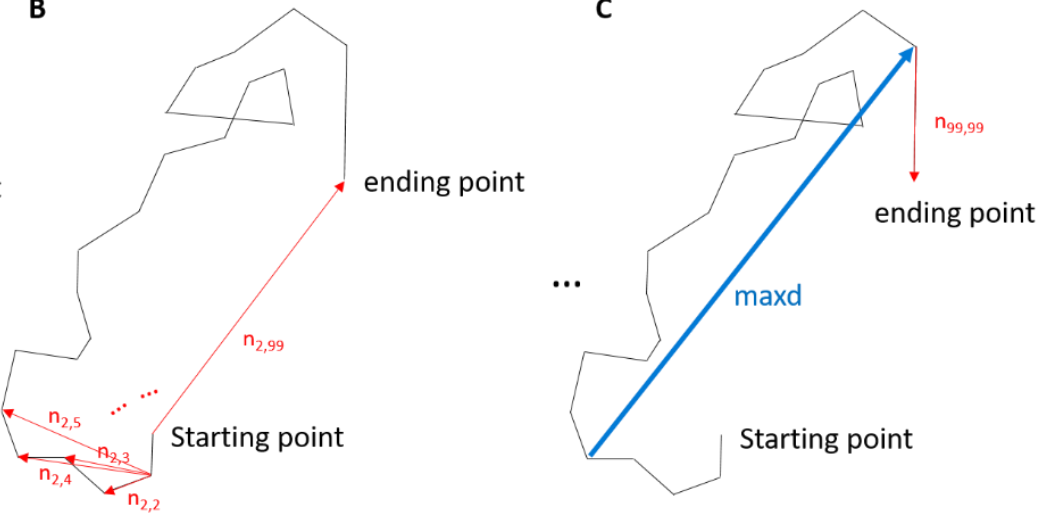

$\operatorname{maxd}_{9 g}=$ Maximum $\left(\mathrm{n}_{99,99}\right)$

$\operatorname{maxd}=$ Maximum $\left(\operatorname{maxd}_{1}, \operatorname{maxd}_{2}, \ldots, \operatorname{maxd}_{g g}\right)$

Figure S2. Definition of the maximum displacement. (A) The maximum displacement from the starting point (\#1) of a sample LD trajectory. (B) The maximum displacement from the second (\#2) time point of the trajectory. (C) The maximum displacement from the second-last (\#99) time point of the trajectory, and the definition of the maxd of the trajectory. 


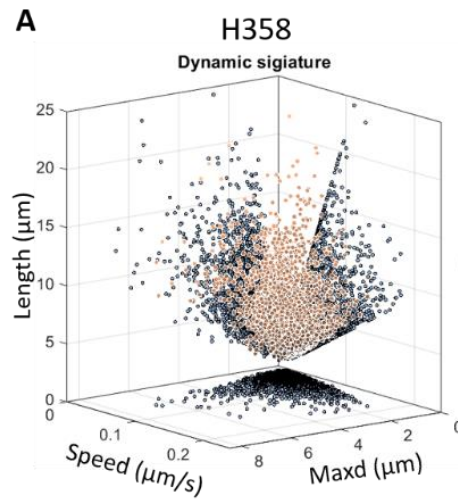

D

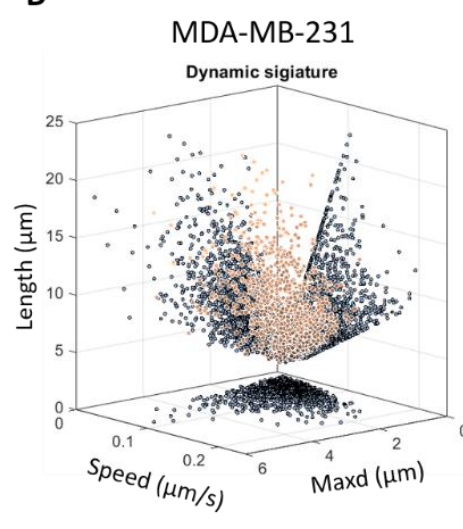

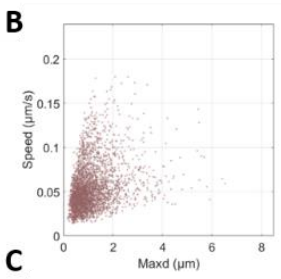
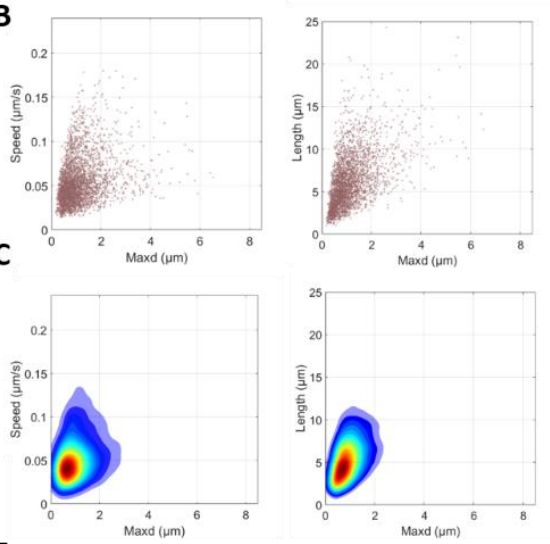

E
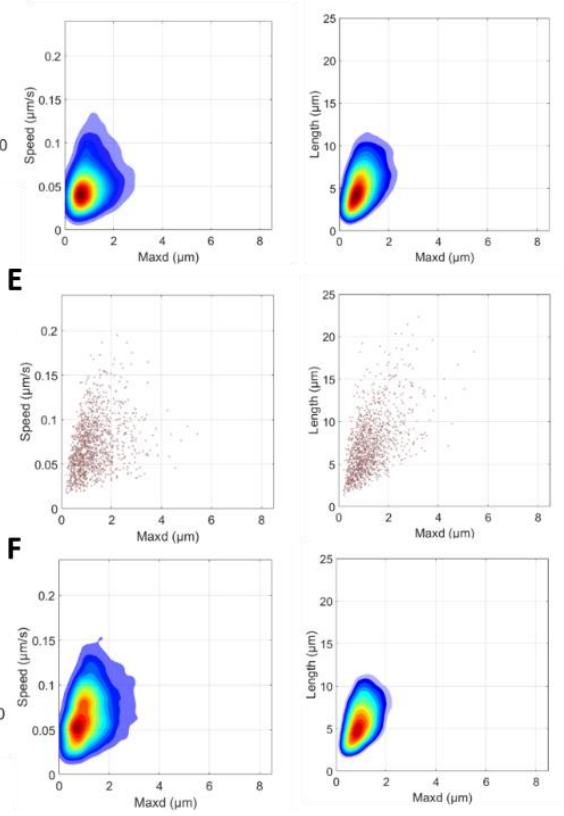
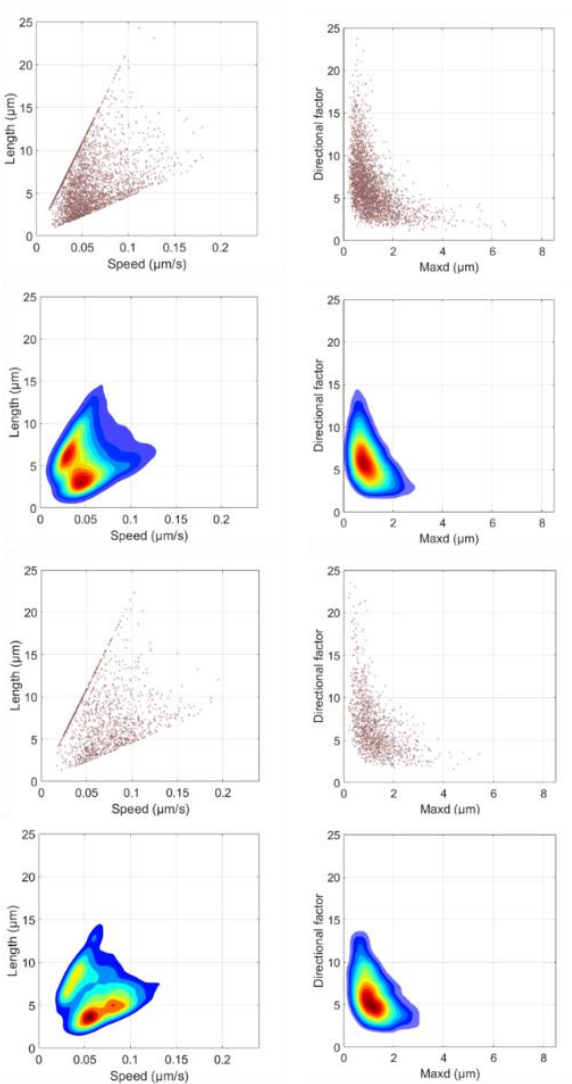

Figure S3. Dynamic signatures of different cell lines. (A) 3D dynamic signature of H358 cells and projections. (B) and (C), scatter and contour plots of $\mathrm{H} 358$ dynamic signatures projected onto the speed-maxd, length-maxd, length-speed, and DF-maxd planes. (D) 3D dynamic signature of MDA-MB-231 cells and projections. $(E)$ and $(F)$, scatter and contour plots of MDA-MB-231 dynamic signatures projected onto the four 2D planes as in panels $(B)$ and $(C)$. 
A

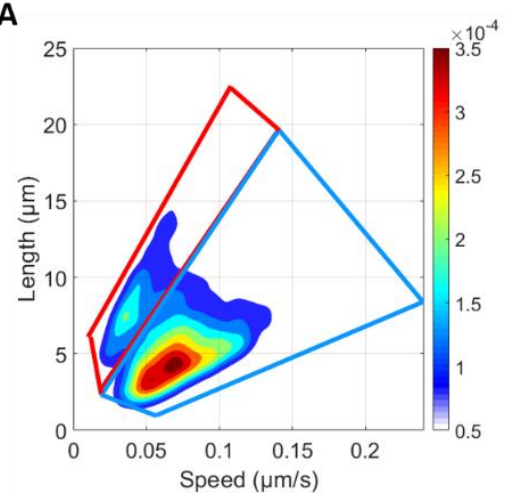

B

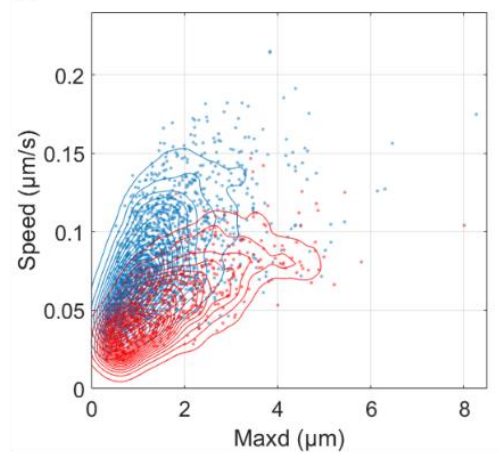

C

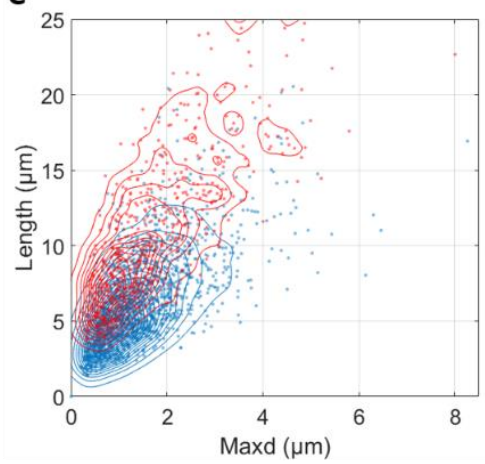

Figure S4. Subpopulation analysis from the length-speed domain. (A) Two gates of the subpopulation of LDs based on the density plot of their trajectories in the length-speed domain. (B) and $(C)$ The density plot of the two subpopulations in the speed-maxd and the length-maxd planes, respectively.
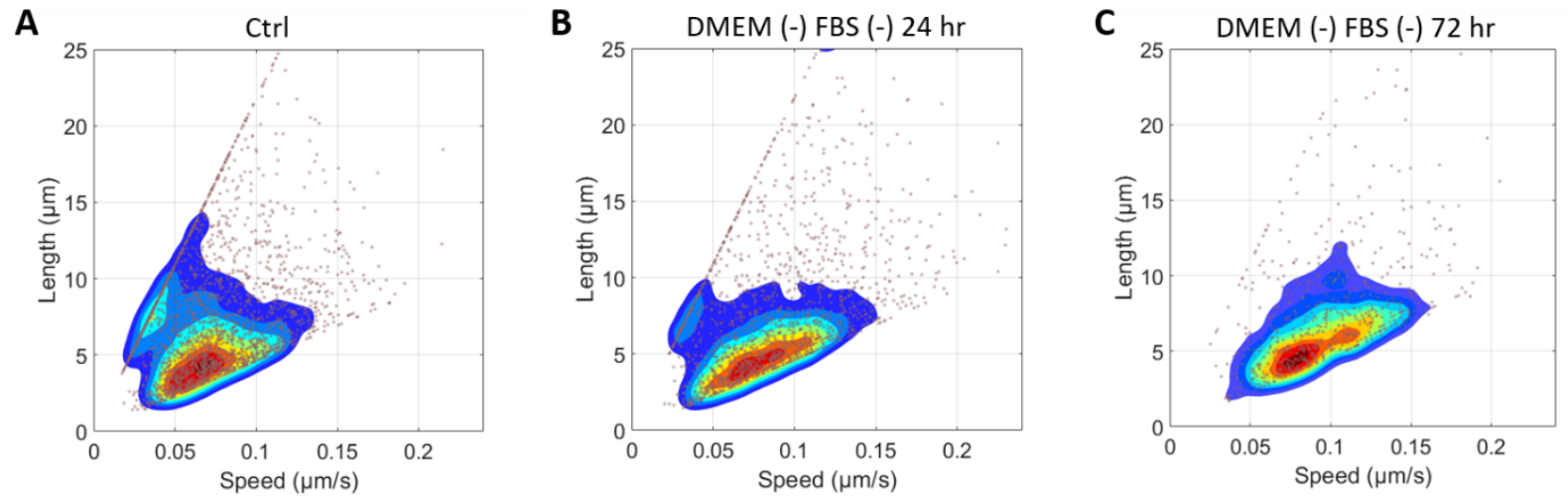

Figure S5. Dynamic signatures of MIAPaCa-2 cells in the length-speed domain in starved conditions. Density plots of the LD trajectories in the (A) control $\left(37^{\circ} \mathrm{C}\right.$, normal culture medium), (B) $24 \mathrm{hr}$ starvation, and (C) $72 \mathrm{hr}$ starvation conditions. 
A

Ctrl
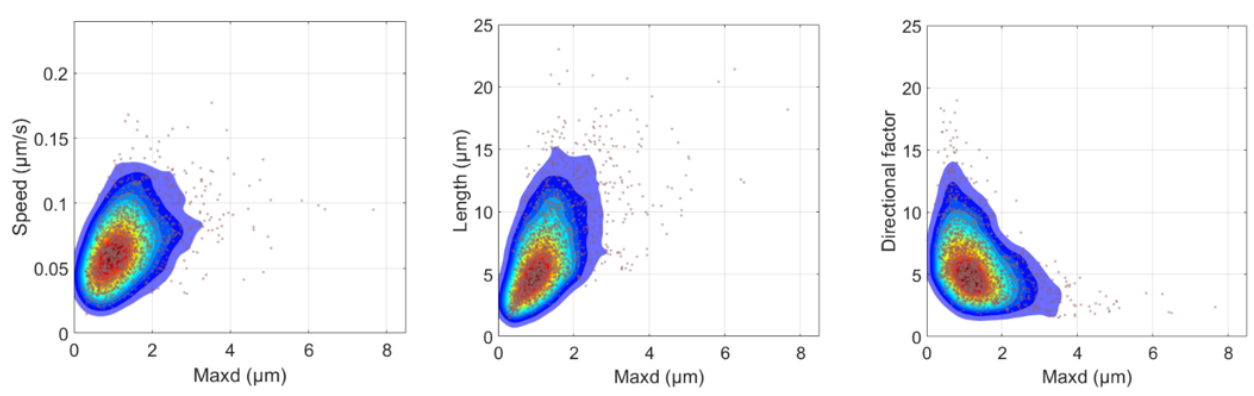

B
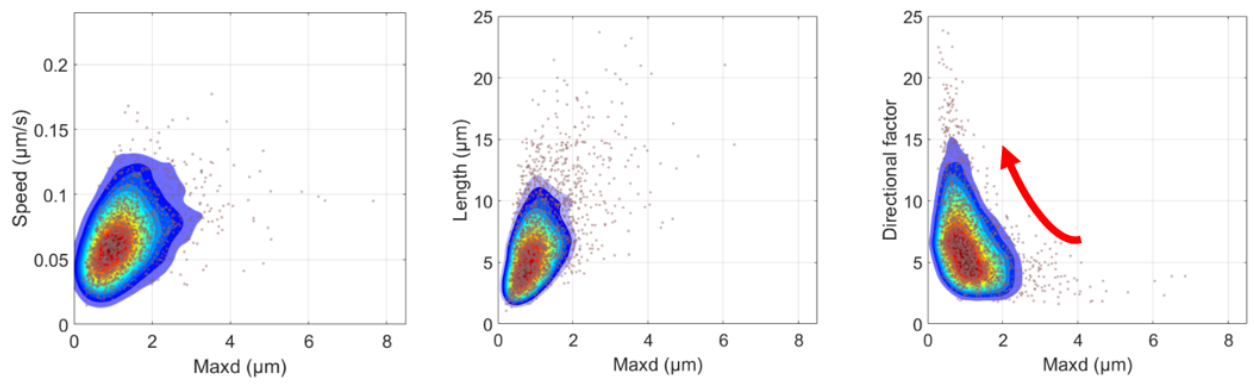

C
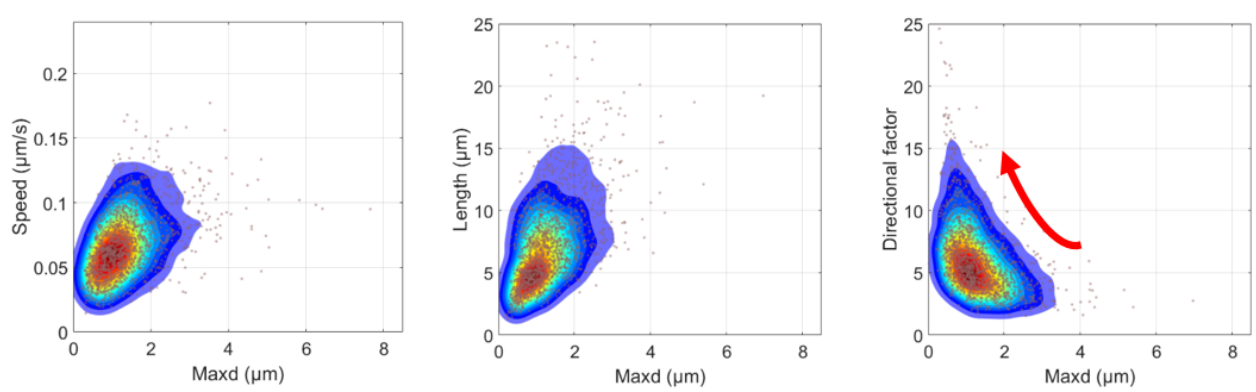

D

BODIPY

$4 \mu \mathrm{g} / \mathrm{mL} 1 \mathrm{hr}$

BODIPY

$2 \mu \mathrm{g} / \mathrm{mL} 6 \mathrm{hr}$
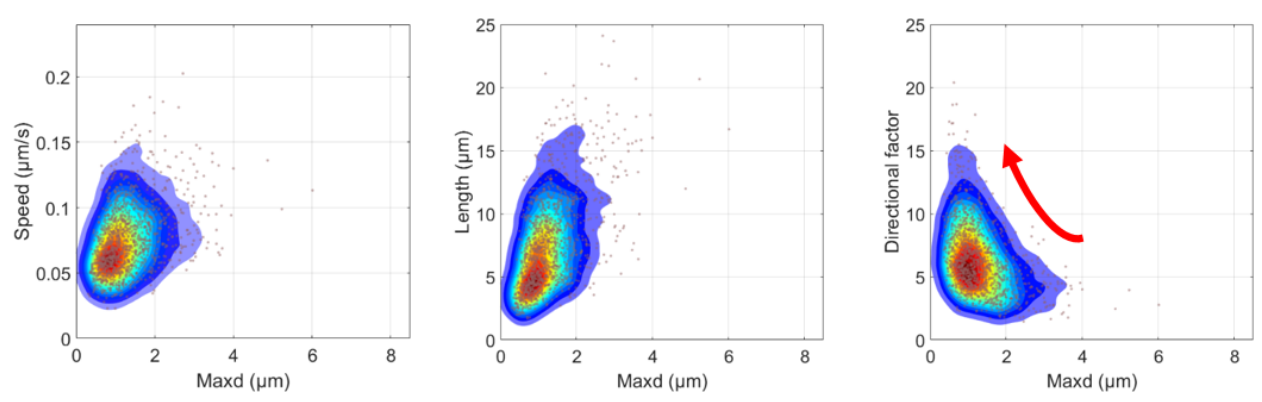

Figure S6. The effects of BODIPY labeling on LD dynamics. (A) The dynamic signature of MIAPaCa-2 cells in the normal condition projected onto the speed-maxd, length-maxd, and DFmaxd planes. (B)-(D) The dynamic signatures of MIAPaCa-2 treated with $2 \mu \mathrm{g} / \mathrm{mL}$ BODIPY for $1 \mathrm{hr}, 4 \mu \mathrm{g} / \mathrm{mL}$ BODIPY for $1 \mathrm{hr}$, and $2 \mu \mathrm{g} / \mathrm{mL}$ BODIPY for $6 \mathrm{hr}$ projected on the three planes as in panel $(A)$. 
A

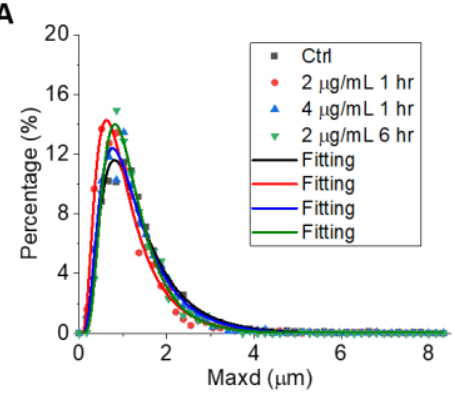

C

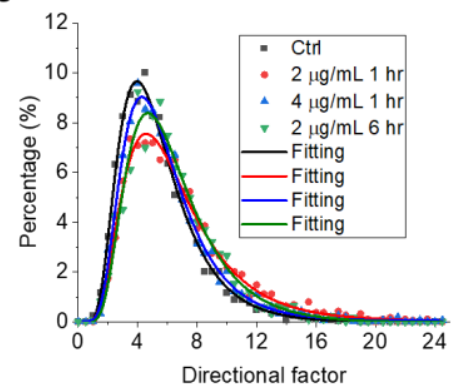

E

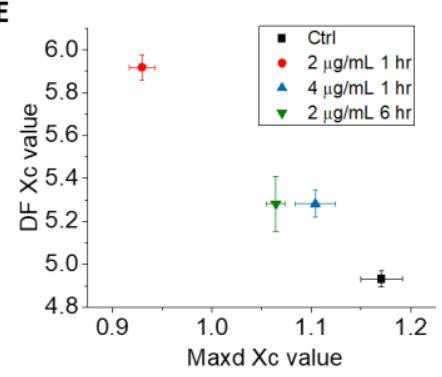

B
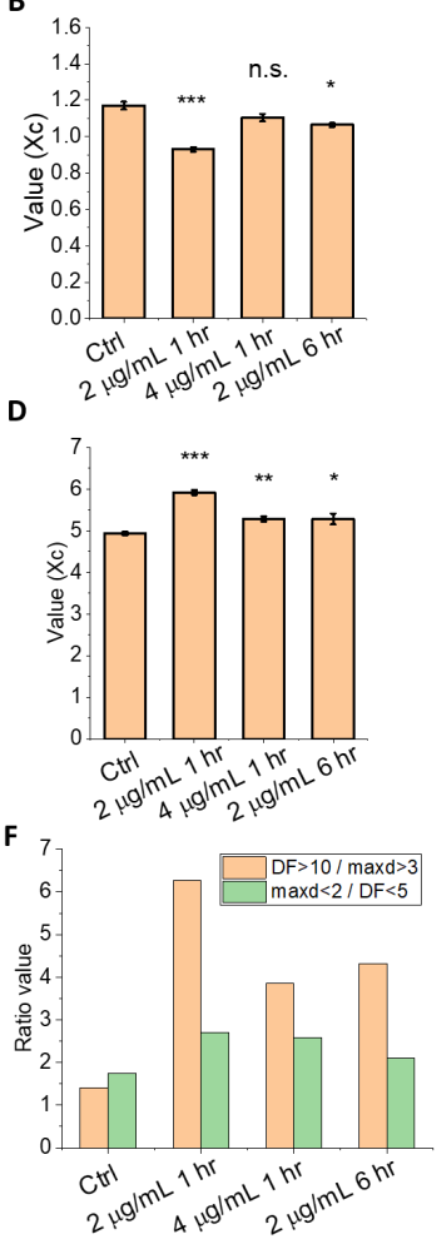

Figure S7. Quantification of the effects of BODIPY labeling on LD dynamics. (A) Histograms (dots) and the lognormal fitting of the histograms (solid curves) of MIAPaCa-2 cells at the maxd domain for the control and the three BODIPY treated groups. (B) $X_{c}$ values derived from the histogram fitting in panel (A). (C) Histograms (dots) and the lognormal fitting of the histograms (solid curves) of MIAPaCa-2 cells in the DF domain. (D) $X_{C}$ values derived from the histogram fitting in panel (C). (E) $X c$ values of the four conditions plotted on the DF-maxd plane. (F) The population ratio of $D F>10$ / maxd $>3$ LDs (yellow) and maxd $<2$ / $D F<5$ LDs (green) for the four experimental conditions. 
A

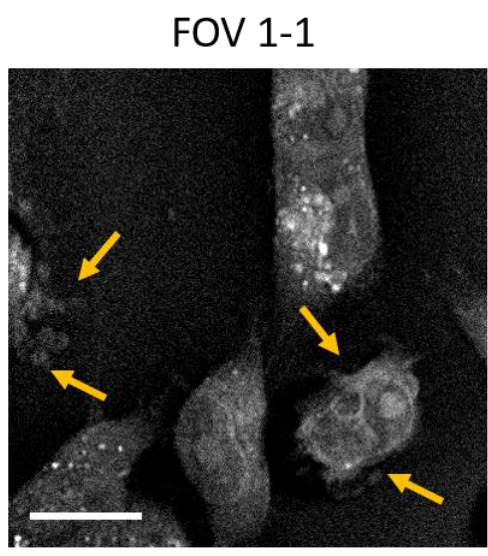

B

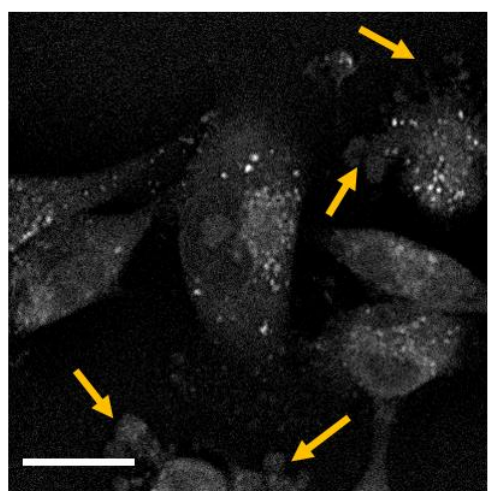

MIAPaCa-2 $2 \mu \mathrm{g} / \mathrm{mL} 6 \mathrm{hr}$

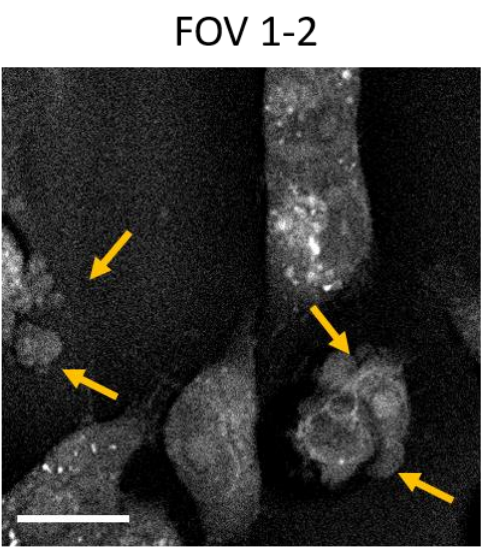

FOV 2-2

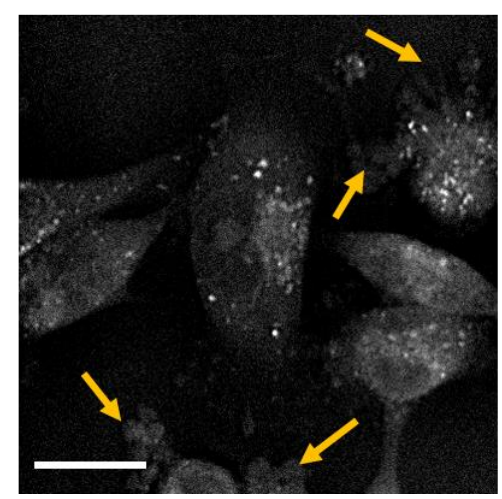

FOV 1-3

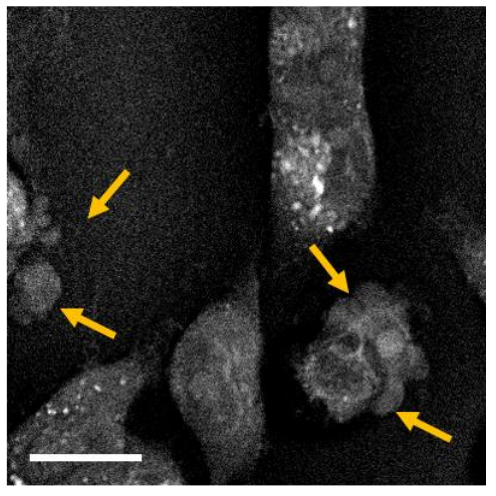

FOV 2-3

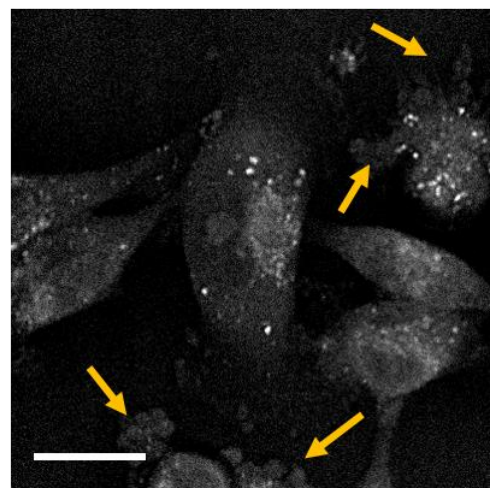

Figure S8. Impact of $2 \mu \mathrm{g} / \mathrm{mL}$ BODIPY on MIAPaCa-2 cells. (A) CARS images from three timepoints for MIAPaCa-2 cells labeled with $2 \mu \mathrm{g} / \mathrm{mL}$ BODIPY for $6 \mathrm{hr}$. The time interval between the two adjacent images is $110 \mathrm{~s}$. (B) Another field of view selected similarly as panel (A). Arrows point out cell membrane blebbing areas. Scale bars: $20 \mu \mathrm{m}$. 
A

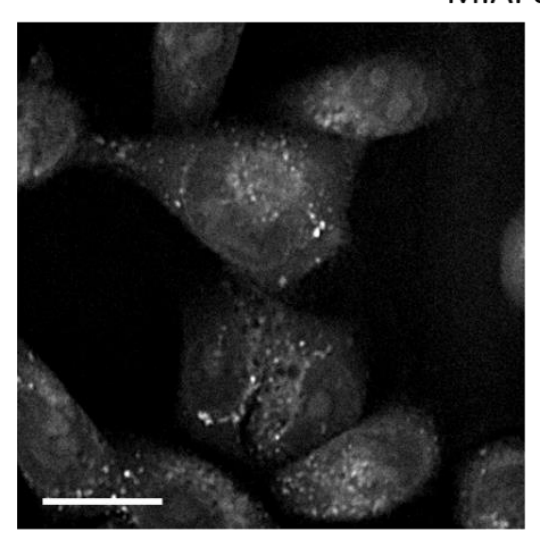

B

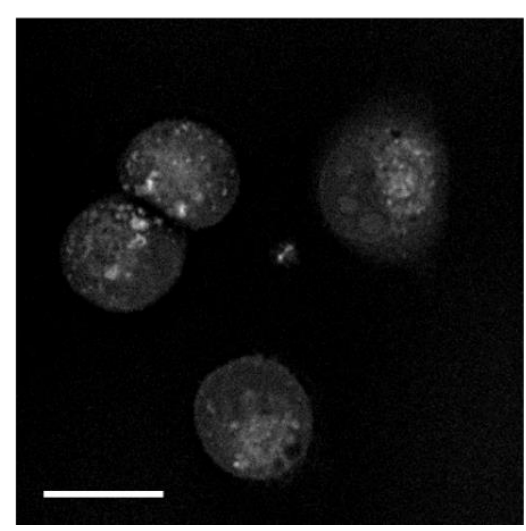

MIAPaCa-2 ctrl

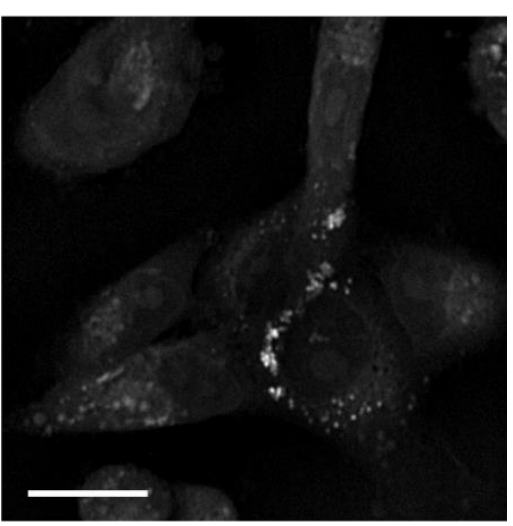

MIA PaCa-2 $4 \mu \mathrm{g} / \mathrm{mL}$ BODIPY $1 \mathrm{hr}$

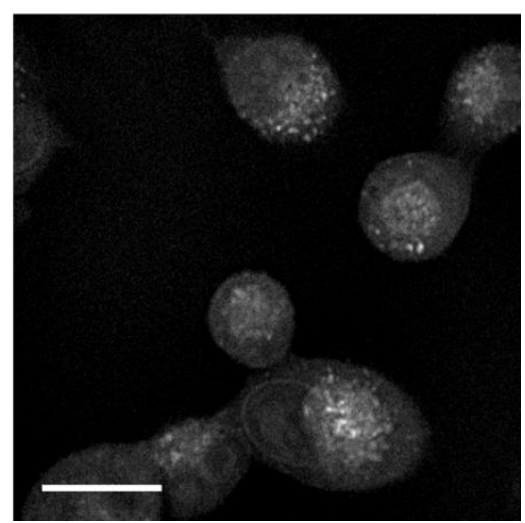

Figure S9. Impact of $4 \mu \mathrm{g} / \mathrm{mL}$ BODIPY on MIAPaCa-2 cells. (A) CARS images from MIAPaCa2 cells without BODIPY labeling. (B) CARS images from MIAPaCa-2 cells labeled with $4 \mu \mathrm{g} / \mathrm{mL}$ BODIPY for $1 \mathrm{hr}$. Scale bars: $20 \mu \mathrm{m}$. 
A

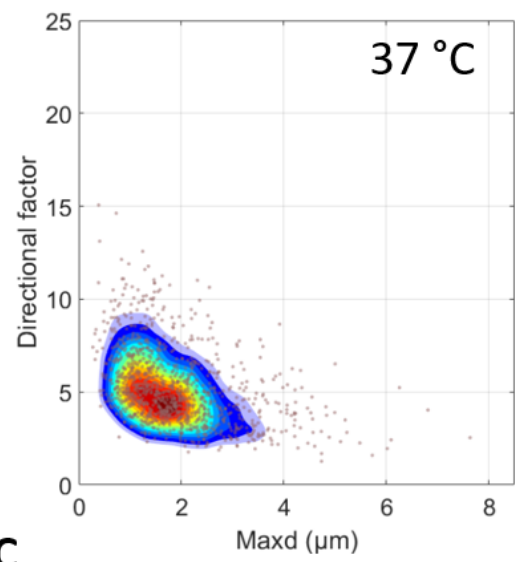

C

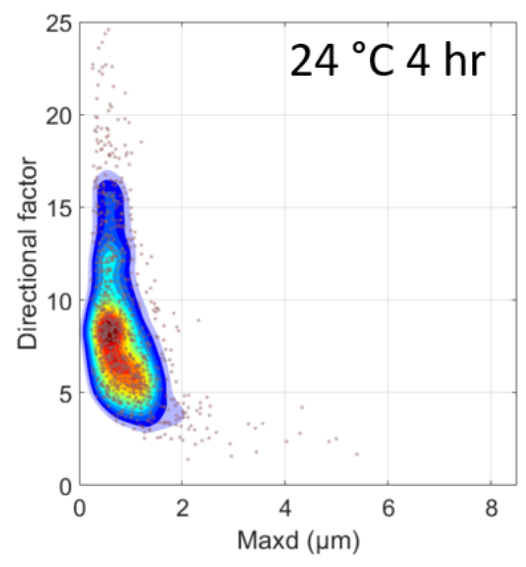

B

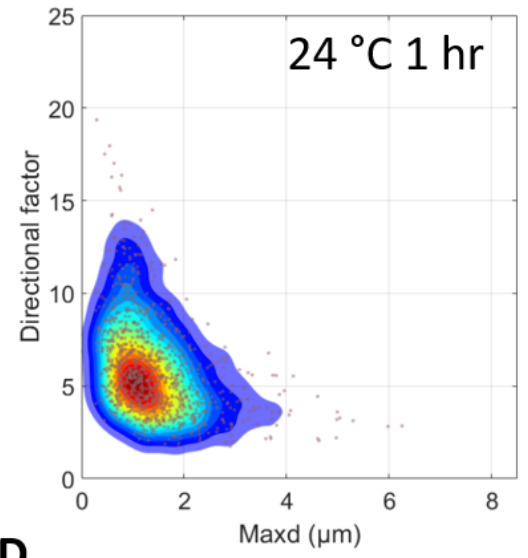

D

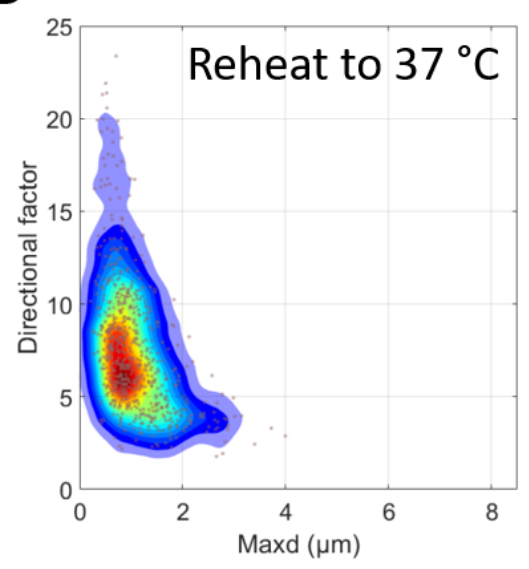

Figure S10. Long exposure to hypothermia caused irreversible changes in LD dynamics. (A)-(D) Dynamic signatures of MIAPaCa-2 cells at $37^{\circ} \mathrm{C}$, hypothermia exposure to $24^{\circ} \mathrm{C}$ for $1 \mathrm{hr}$, hypothermia exposure to $24^{\circ} \mathrm{C}$ for $4 \mathrm{hr}$, and reheat sample to $37^{\circ} \mathrm{C}$, projected onto the $D F$-maxd plane, respectively. 
A
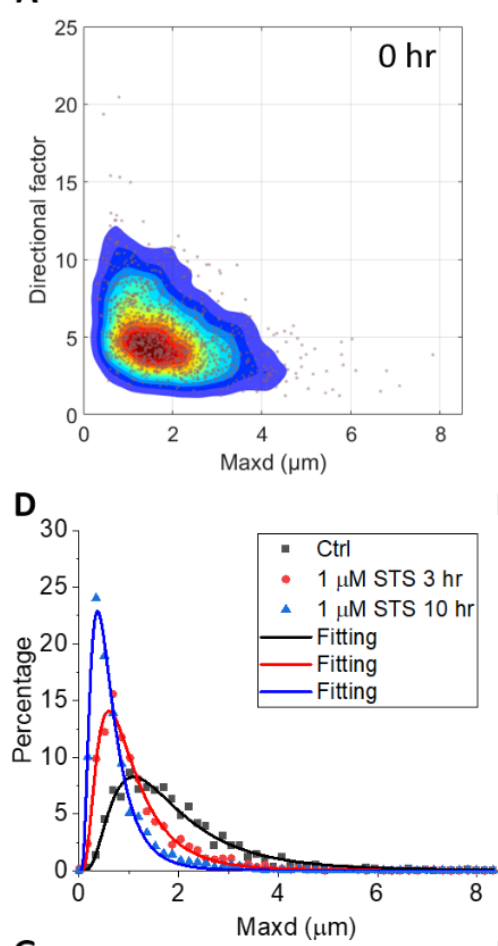

G

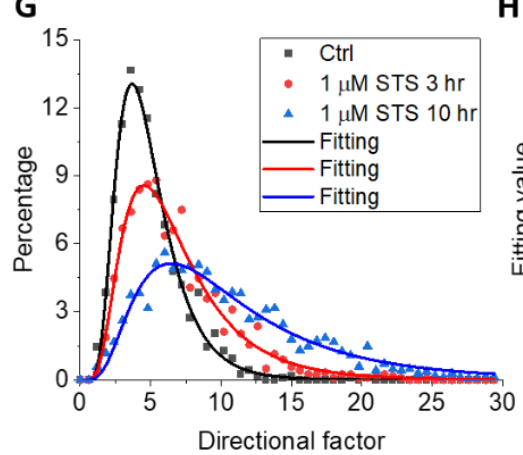

B

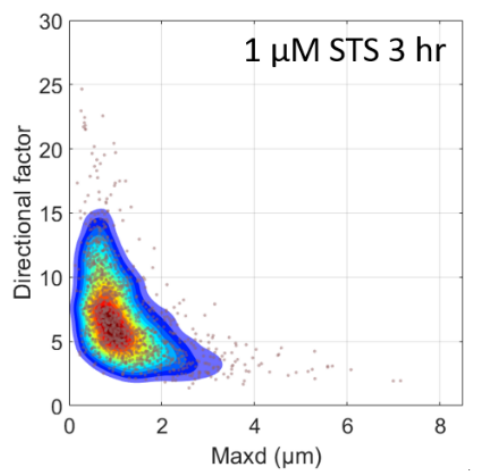

E

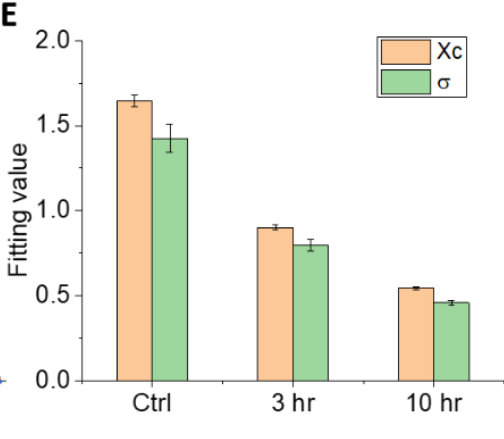

H

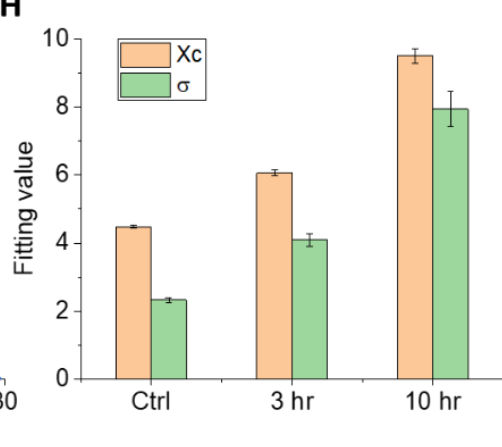

C

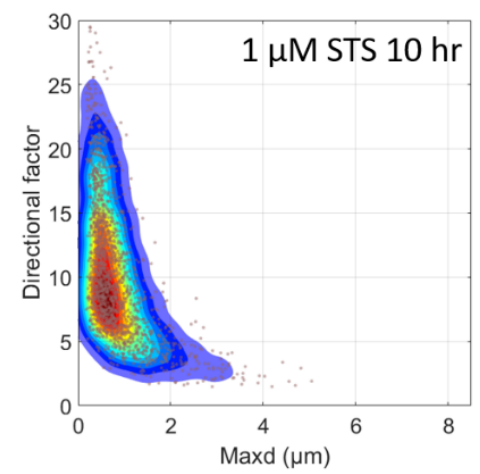

$\mathbf{F}$

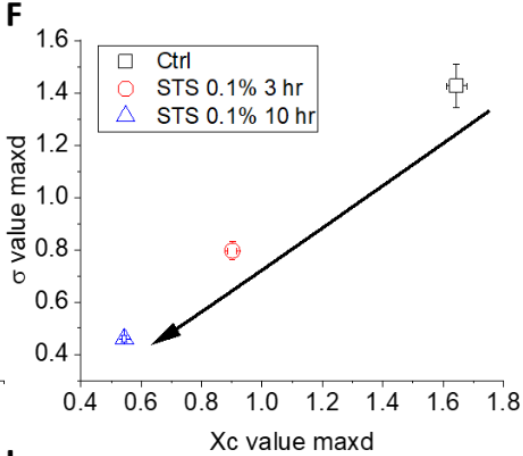

I

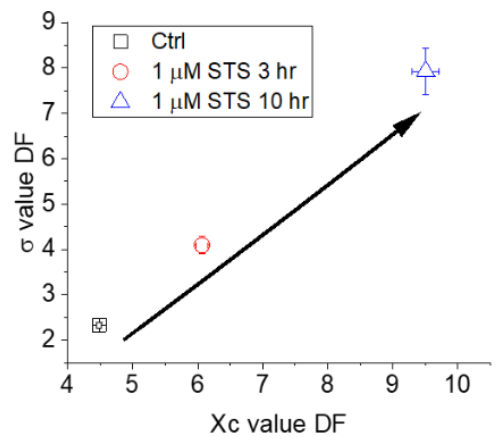

Figure S11. Changes of LD dynamics during apoptosis induced by $1 \mu \mathrm{M}$ STS. (A)-(C) Dynamic signatures of MIAPaCa-2 control group and $1 \mu \mathrm{M}$ STS treatment for $3 \mathrm{hr}$ and $10 \mathrm{hr}$, respectively, projected onto the DF-maxd plane. (D) Histograms (dots) and the lognormal fitting of the histograms (solid curves) of MIAPaCa-2 cells at maxd domain from the control (black), $3 \mathrm{hr}$ STS treatment (red), and $10 \mathrm{hr}$ STS treatment (blue). (E) $X_{c}$ and $\sigma$ values derived from the lognormal fitting from each condition in panel (D). (F) Time-dependent STS treatment results plotted in the maxd $X_{c}-\sigma$ domain. The arrow indicates the change over STS treatment time. $(\mathrm{G})$ Histograms (dots) and the lognormal fitting of the histograms (solid curves) of MIAPaCa-2 cells at DF domain from the control (black), $3 \mathrm{hr}$ STS treatment (red), and $10 \mathrm{hr}$ STS treatment (blue). $(\mathrm{H}) \mathrm{Xc}$ and $\sigma$ values derived from the lognormal fitting from each condition in panel (G). (I) Timedependent STS treatment results plotted in the $D F X_{C}-\sigma$ domain. The arrow indicates the change over STS treatment time. 

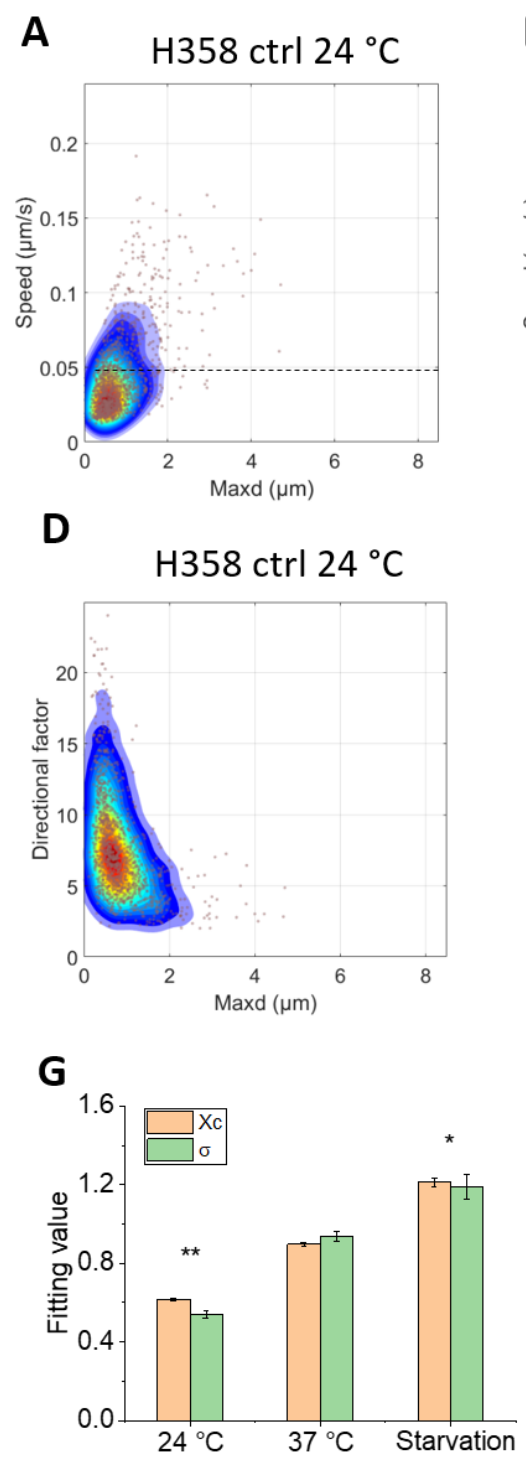

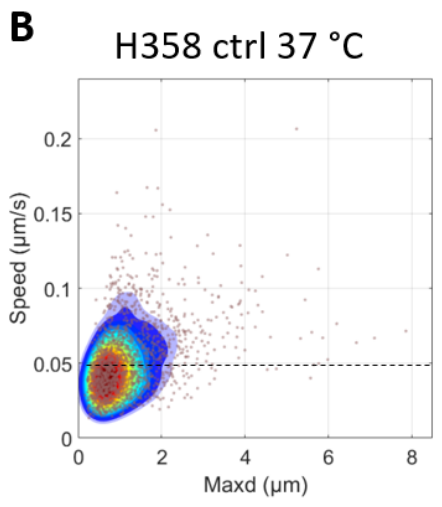

$\mathbf{E}$
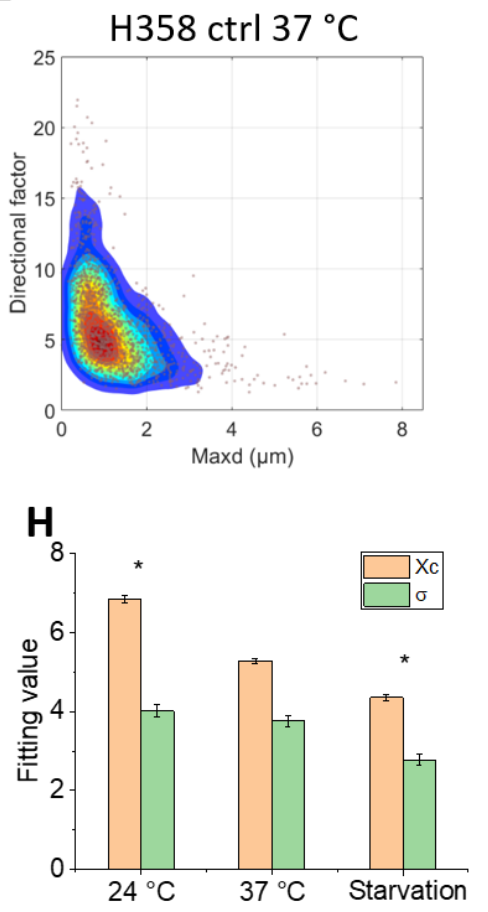

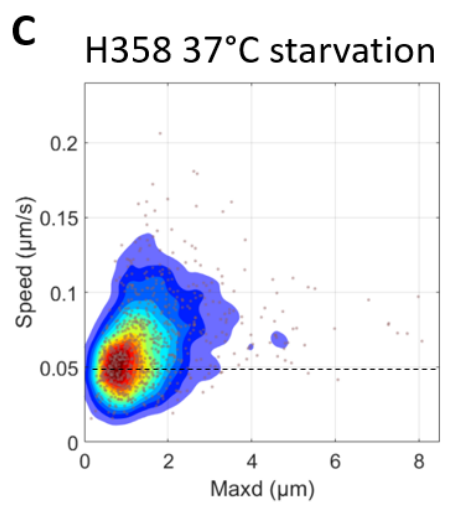

$\mathbf{F}$
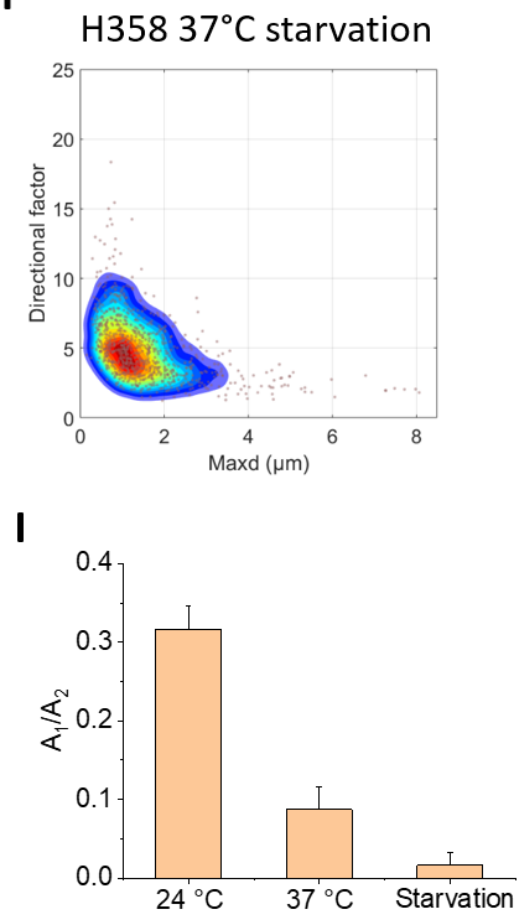

Figure S12. The dynamic signatures of $\mathrm{H} 358$ cells under hypothermia exposure and starvation conditions. (A)-(C) Dynamic signatures of $\mathrm{H} 358$ cells projected onto the speed-maxd plane during $24^{\circ} \mathrm{C}$ hypothermia exposure for $1 \mathrm{hr}$, at normal culture condition, and after $24 \mathrm{hr}$ starvation without glucose and FBS in the culture medium, respectively. (D)-(F) Dynamic signatures of $\mathrm{H} 358$ cells in the same three conditions as in (A)-(C), projected onto the DF-maxd plane. (G) $X c$ and $\sigma$ values derived from the lognormal fitting of maxd from each condition in panels $(\mathrm{A})-(\mathrm{C}) .(\mathrm{H}) X c$ and $\sigma$ values derived from the lognormal fitting of $D F$ from each condition in panels $(D)-(F)$. (I) The ratio of amplitudes $A_{1} / A_{2}$ derived from the dual-lognormal fitting of the speed histograms from the three conditions. 

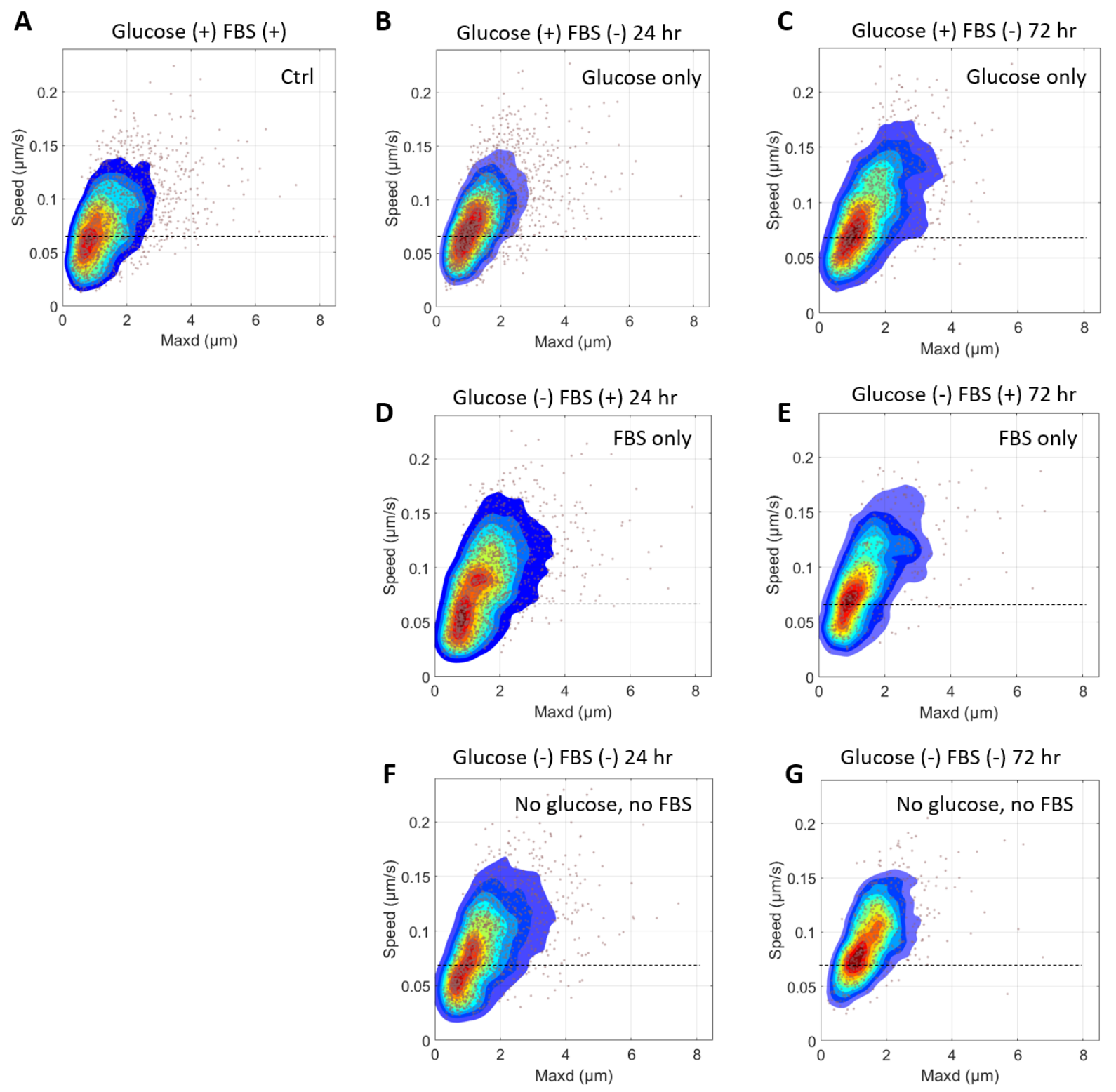

Figure S13. LD dynamic signatures in different starvation conditions. Dynamic signatures of MIAPaCa-2 cells projected onto the speed-maxd plane for (A) the control group; (B) and (C) with only glucose for 24 and $72 \mathrm{hr}$, respectively; (D) and (E) with only FBS for 24 and $72 \mathrm{hr}$, respectively; (F) and (G) with no glucose and no FBS for 24 and $72 \mathrm{hr}$, respectively. The dotted lines are shown as reference marks. 


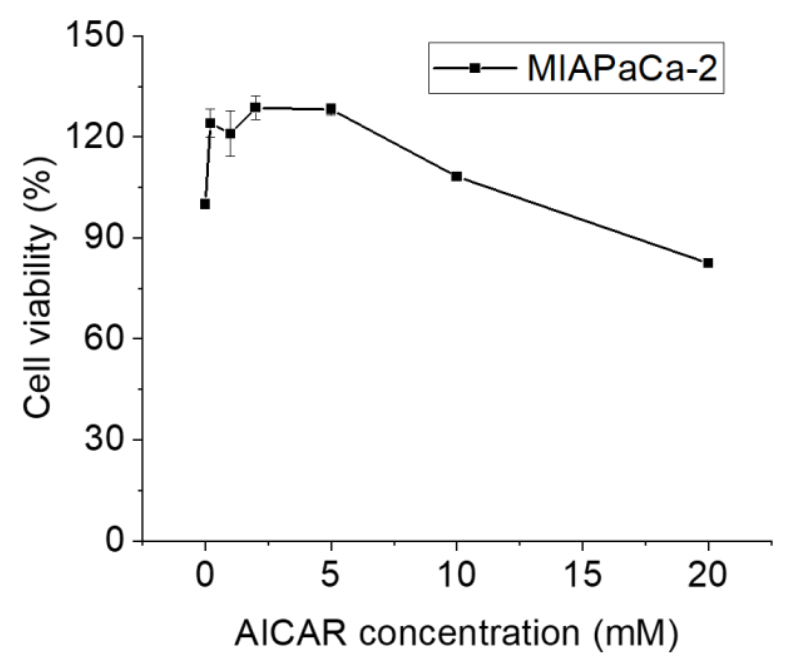

Figure S14. MTT viability assay of MIAPaCa-2 cells treated by AICAR (24 hr). MTT viability results for MIAPaCa-2 cells treated with various concentrations of AICAR in normal culture media for $24 \mathrm{hr}$. The viability was normalized with the control group ( $0 \mathrm{mM}$ AICAR). $\mathrm{n}=6$. 
A
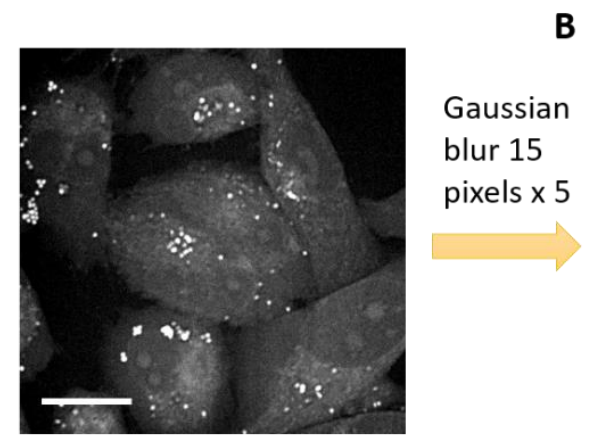

B

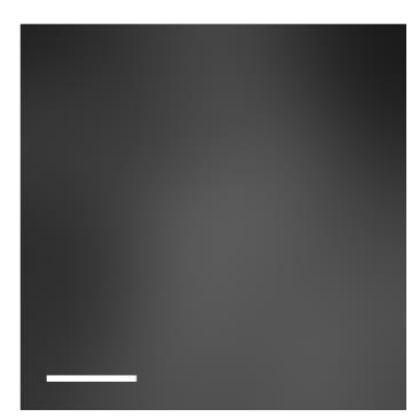

Original image /

Gaussian

blur image

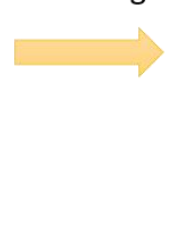

Particle analysis $<100$ pixels

D

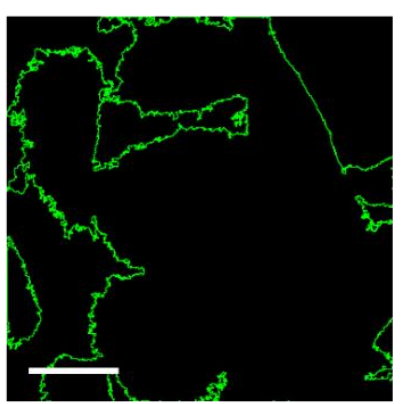

E

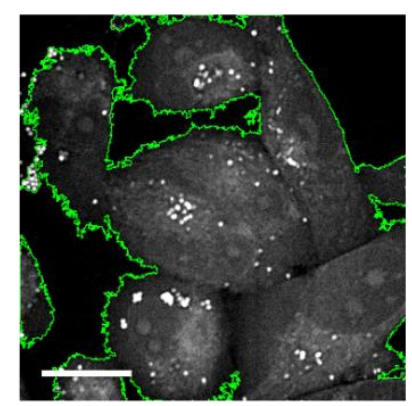

Cell outline

Cell outline

overlay

$A=$ Cell area

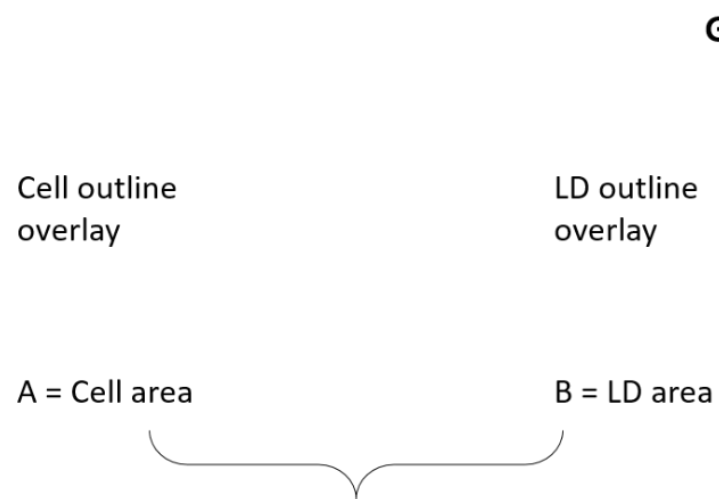

LD area $\%=B / A \times 100 \%$
C

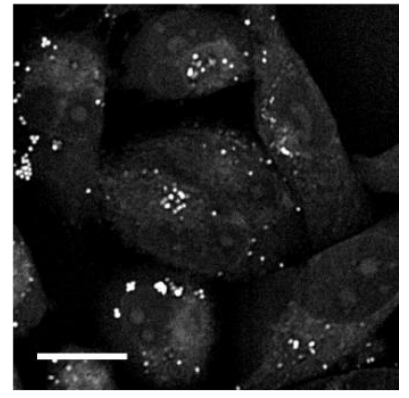

$\mathbf{F}$

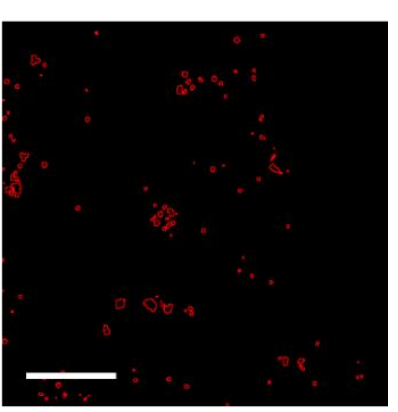

G

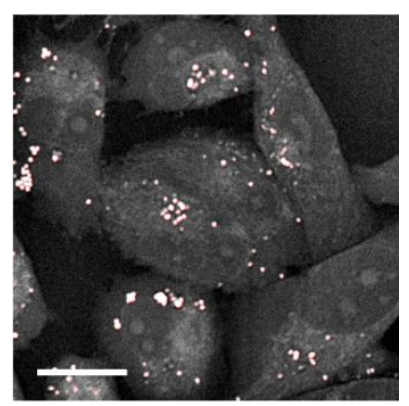

Figure S15. Quantitative analysis of LD area percentage. (A) The original CARS image. (B) Gaussian-blurred image using 15 pixels for 5 times. (C) Image obtained by dividing the original CARS image by the Gaussian-blurred image. (D) Particle tracking after intensity thresholding of panel $(A)$ using particle size $>1000$ pixels delineates the outlines of cells. (E) Superposition of image in panel (A) and the outlines in panel (D). (F) Particle tracking after intensity thresholding of panel $(C)$ using particle size $<100$ pixels outlines the LD particles. (G) Superposition of image in panel $(A)$ and the outlines in panel $(F)$. The total areas $A$ for cells and $B$ for LDs are calculated. The LD area \% is defined as B/A x 100\%. Scale bars: $20 \mu \mathrm{m}$. 
A
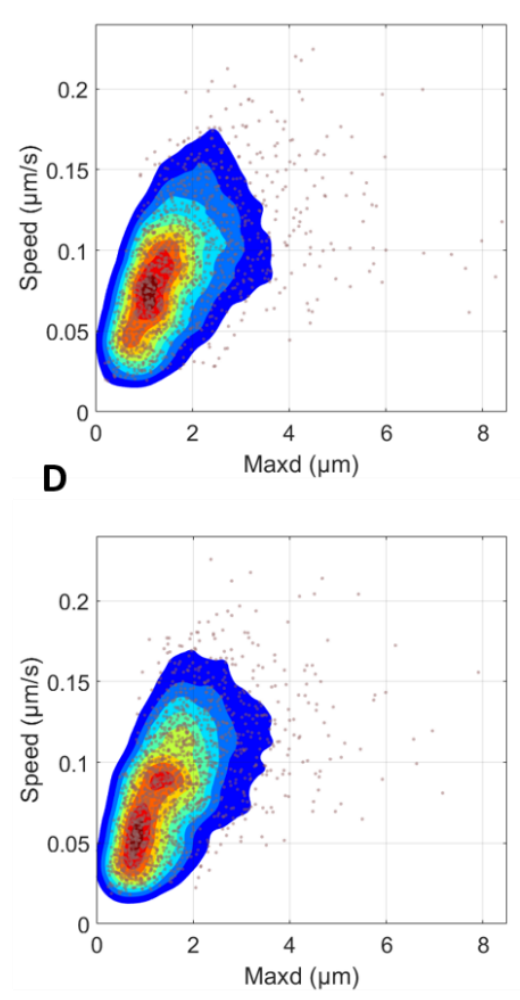

G

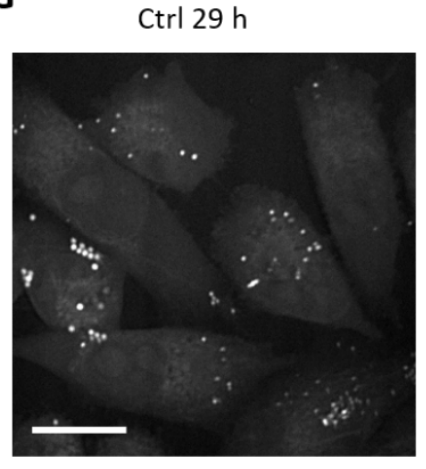

B
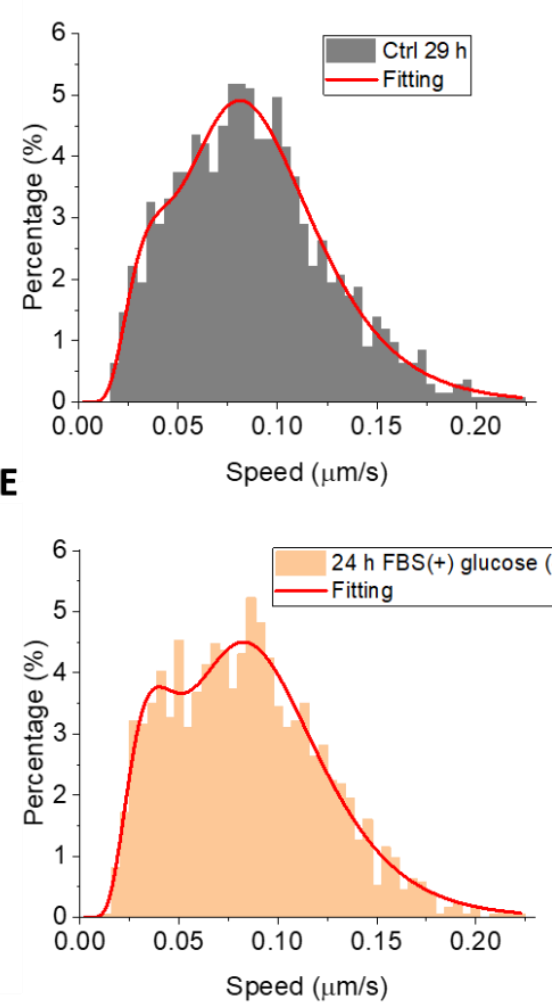

H

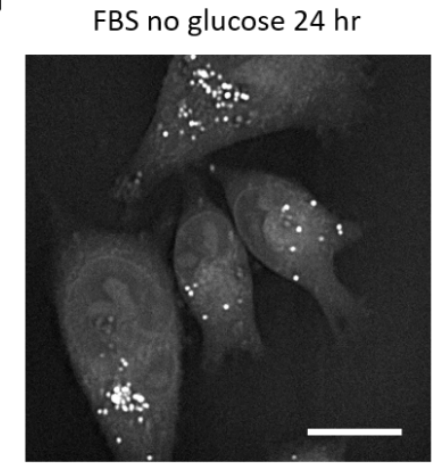

C
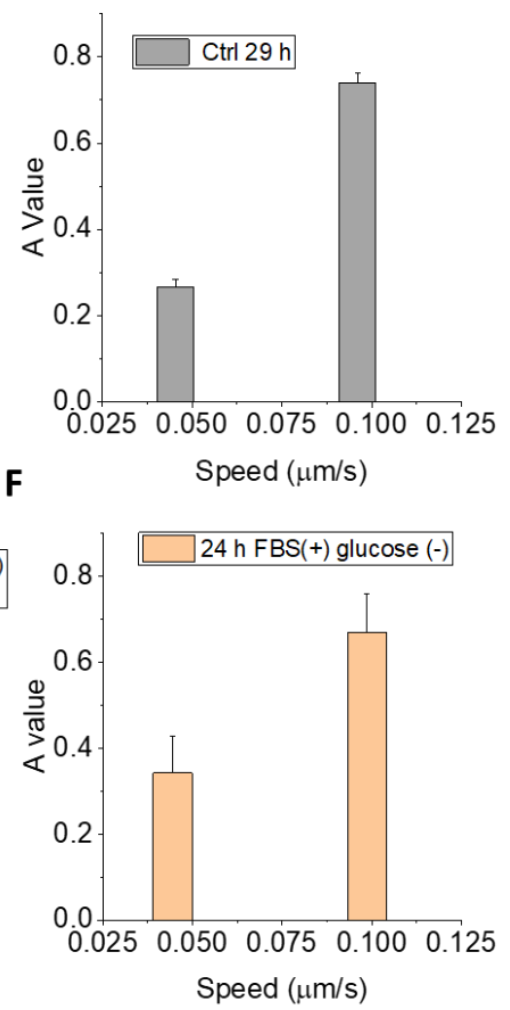

I

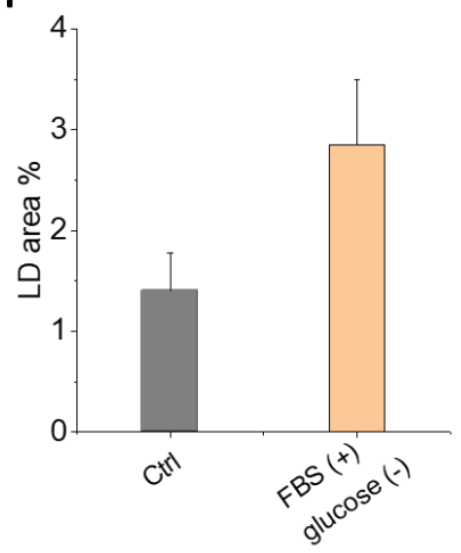

Figure S16. The changes of dynamic signatures and LDs of MIAPaCa-2 cells with only FBS as the energy source. (A) The dynamic signature of MIAPaCa-2 cells projected onto the speedmaxd plane for the control group. (B) The histogram (bars) in the speed domain and the duallognormal function fitting (solid curve) for the control group. (C) Amplitude values as a function of $X c$ derived from the histogram fitting in panel (B). (D) The dynamic signature of MIAPaCa-2 cells projected onto the speed-maxd plane after $24 \mathrm{hr}$ culture with FBS and glucose-free DMEM. (E) The histogram (bars) in the speed domain and the dual-lognormal function fitting (solid curve) for the FBS-only cultured group. (F) Amplitude values as a function of $X_{c}$ derived from the histogram fitting in panel $(\mathrm{E})$. $(\mathrm{G})$ and $(\mathrm{H})$, sample CARS images for the control and FBS-only groups, respectively. (I) LD area \% derived from the CARS images using the method described in Figure S15. Scale bars: $20 \mu \mathrm{m}$. 

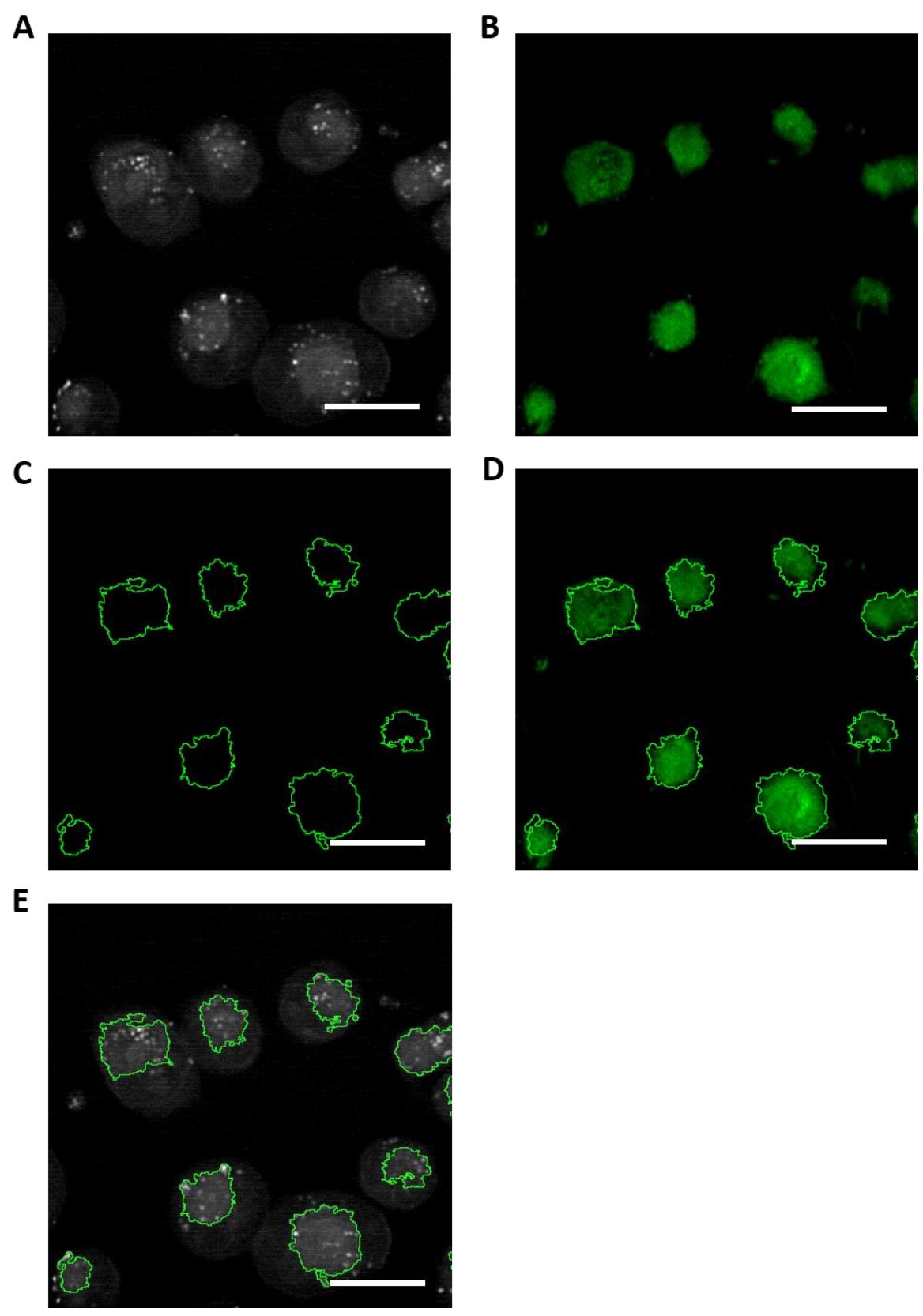

Figure S17. Correlate CARS intensity thresholding with ER Tracker $^{\mathrm{TM}}$ labeling. (A) The original CARS image of MIAPaCa-2 cells. (B) TPEF image of ER from the same field of view in panel (A). The cells were labeled by ER Tracker ${ }^{\mathrm{TM}}$ Green. (C) Intensity thresholding from the original CARS image outlines ER areas. (D) Superposition of label-free CARS-outlined ER area and ER Tracker ${ }^{\mathrm{TM}}$-labeled ER areas. (E) Superposition of the original CARS image with the ER outlines from panel $(\mathrm{C})$. 


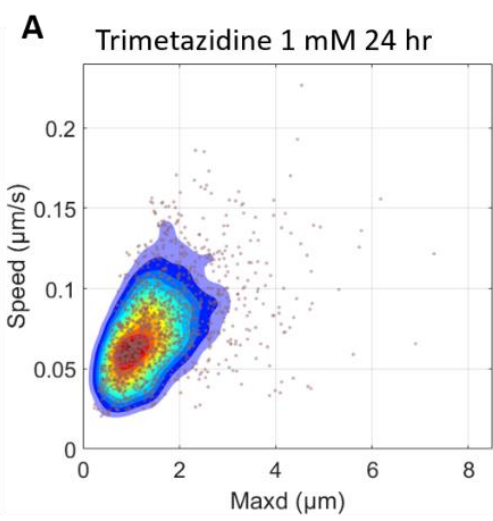

B

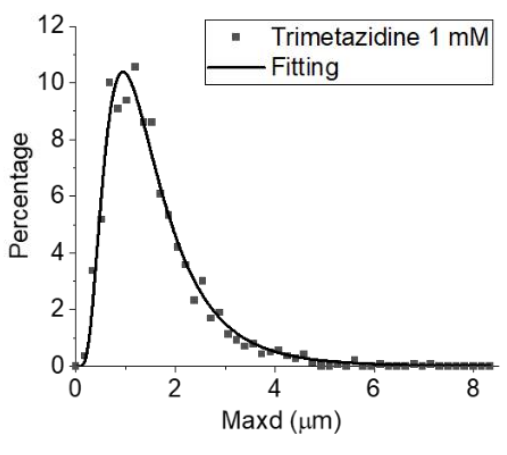

C

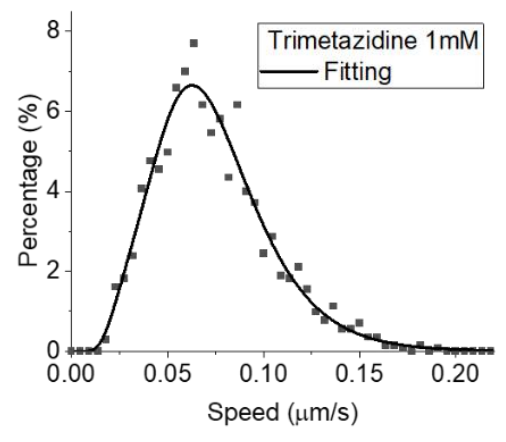

E

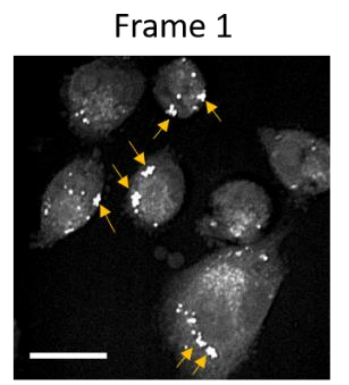

Frame 50

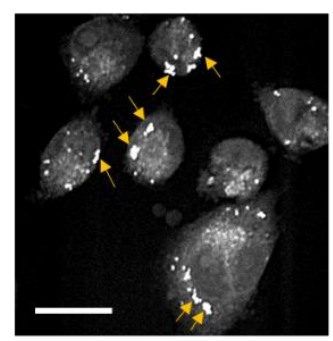

Frame 100

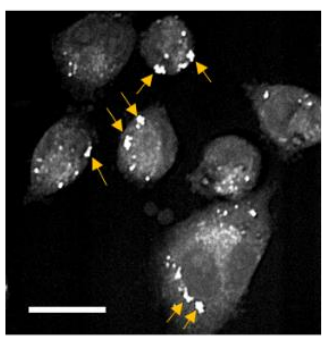

Figure S18. The impact of trimetazidine on LD dynamics. (A) The dynamic signature of MIAPaCa-2 projected onto the speed-maxd plane after $1 \mathrm{mM}$ trimetazidine treatment for $24 \mathrm{hr}$. (B) The histogram (dots) in the maxd domain and the lognormal fitting (solid curve) for the trimetazidine treatment. (C) The histogram (dots) in the speed domain and the dual-lognormal function fitting (solid curve) for the trimetazidine treatment. (D) The dynamic feature of the trimetazidine treatment, together with other treatments, in the $A_{1} / A_{2}-X_{c}$ domain. (E) CARS images for the trimetazidine-treated MIAPaCa-2 cells. The time interval between 1-50 and 50-100 frames are $110 \mathrm{~s}$. Arrows point out LD aggregations in each frame. Scale bars: $20 \mu \mathrm{m}$. 


\section{Supplementary Videos}

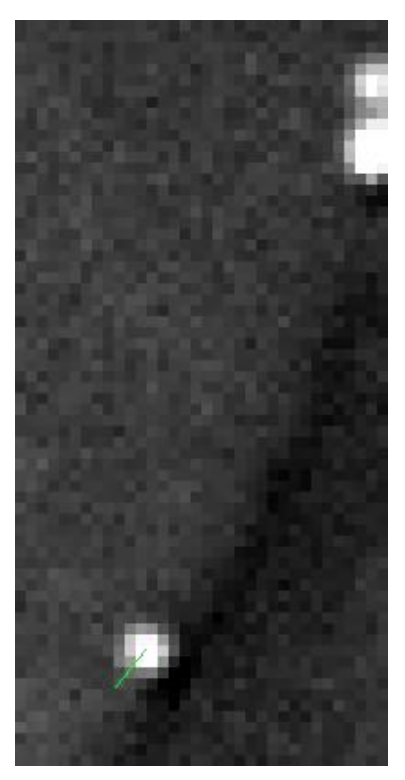

Video S1. Tracing an LD trajectory in a living MIAPaCa2 cell shown in Figure 1C.

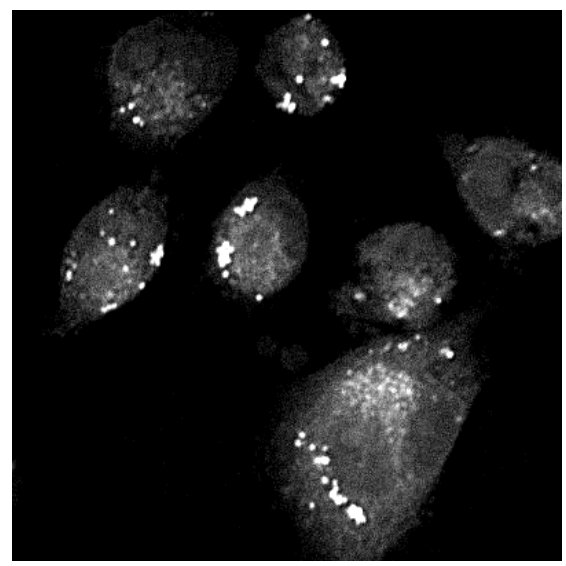

Video S2. Time-lapse CARS images showing LD aggregations in MIAPaCa2 cells treated with $1 \mathrm{mM}$ trimetazidine. 